Os artigos dos Textos para Discussão da Escola de Economia de São Paulo da Fundação Getulio Vargas são de inteira responsabilidade dos autores e não refletem necessariamente a opinião da FGV-EESP. É permitida a reprodução total ou parcial dos artigos, desde que creditada a fonte.

Escola de Economia de São Paulo da Fundação Getulio Vargas FGV-EESP www.eesp.fgv.br 


\title{
Dynamic coordination with timing frictions: theory and applications*
}

\author{
Bernardo Guimaraes ${ }^{\dagger} \quad$ Caio Machado $\quad$ Ana Elisa Pereira $^{\S}$
}

August 2017

\begin{abstract}
We start by presenting the general model of dynamic coordination with timing frictions and some key theoretical results. We prove the model features a unique rationalizable equilibrium, present a method to solve the social planner problem and derive expressions for the equilibrium threshold in limiting cases. With this toolkit in hand, we get analytical results for a case with linear preferences and present several applications, ranging from network externalities to statistical discrimination and to macroeconomics. Besides generating insights for specific questions, the applications illustrate the potential of the model to accommodate a large set of economic problems. Last, we show extensions of the framework that allow for endogenous hazard rates, preemption motives and ex-ante heterogeneous agents.
\end{abstract}

\section{Contents}

1 Introduction

2 The framework $\quad 5$

2.1 Multiple equilibrium benchmark . . . . . . . . . . . . . . . . 6

2.2 Equilibrium uniqueness . . . . . . . . . . . . . . . . . . . 7

2.2.1 Proof of equilibrium uniqueness . . . . . . . . . . . . . . . . 8

2.2.2 Relation to 'global games' . . . . . . . . . . . . . . . . . . . . 11

2.3 The social planner's problem . . . . . . . . . . . . . . . . . . . . . . 12

2.4 Bifurcation probabilities . . . . . . . . . . . . . . . . . . . 13

2.5 Results for limiting cases . . . . . . . . . . . . . . . . . . . . . . . . . . . . 15

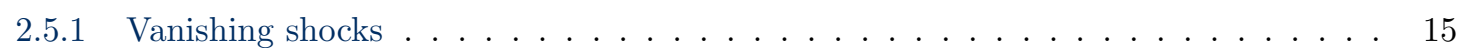

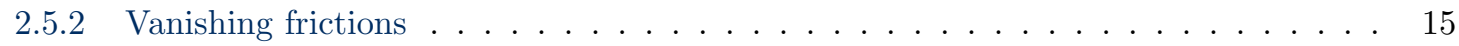

2.6 The planner's problem with vanishing shocks . . . . . . . . . . . . . . . . . 17

${ }^{*}$ We thank the students of the course on Dynamic Coordination at the Sao Paulo School of Economics - FGV for their helpful comments on this material.

†Sao Paulo School of Economics - FGV. Email: bernardo.guimaraes@fgv.br.

${ }^{\ddagger}$ Instituto de Economía, Pontificia Universidad Católica de Chile. Email: caio.machado@uc.cl.

$\S$ School of Business and Economics, Universidad de los Andes, Chile. Email: apereira@uandes.cl. 
3.1 Linear utility . . . . . . . . . . . . . . . . . . . . . . . . 18

3.1.1 Equilibrium threshold in limiting cases . . . . . . . . . . . . . . . . . 19

3.1.2 The planner problem with network externalities . . . . . . . . . . . . . . 19

3.1.3 Symmetric network effects . . . . . . . . . . . . . . . . . . . 20

3.1.4 Asymmetric network effects . . . . . . . . . . . . . . . . . 22

3.1.5 Alternative interpretation: negative externalities . . . . . . . . . . . . . 23

3.2 Statistical discrimination . . . . . . . . . . . . . . . . . . . . . 23

3.2 .1 Equilibrium . . . . . . . . . . . . . . . . . . . . 24

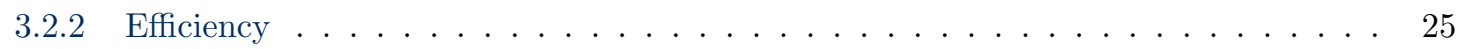

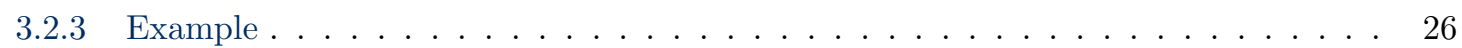

3.3 A macroeconomic model . . . . . . . . . . . . . . . . . . . . . . . 27

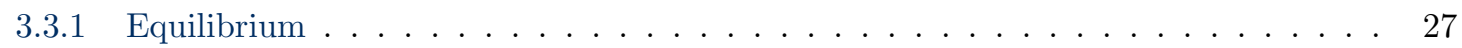

3.3.2 Optimal stimulus policies . . . . . . . . . . . . . . . . . 29

3.3.3 Optimal stimulus with no shocks . . . . . . . . . . . . . . . . 31

3.3.4 Numerical solution . . . . . . . . . . . . . . . . . . . . . 32

3.4 Housing and coordination . . . . . . . . . . . . . . . . . . . . 35

4 Extensions $\quad 36$

4.1 Endogenous timing . . . . . . . . . . . . . . . . . . . . . . . . . . . . . . . . . . . . . . . .

4.1 The planner's problem . . . . . . . . . . . . . . . . . . . 38

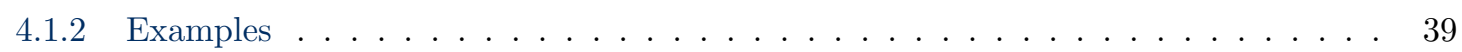

4.2 Ex ante heterogeneous agents . . . . . . . . . . . . . . . . . . 39

4.2 .1 Bifurcation probabilities . . . . . . . . . . . . . . . 41

4.2 .2 Results for limiting cases _. . . . . . . . . . . . . . . . . . 42

4.3 Coordination and preemption . . . . . . . . . . . . . . . . . 45

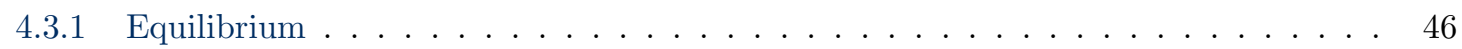

$\begin{array}{lr}\text { References } & 48\end{array}$ 


\section{Introduction}

Several economic problems exhibit strategic complementarities: one's payoff from some action is larger if others take this action as well. For example, in a scenario of bank runs, withdrawing deposits from the bank might be the optimal action only if others also do so; the decision about joining and posting on Facebook depends crucially on whether other people are doing the same; for firms considering whether to invest or not, one important factor is the demand for their goods, which in turn depends on whether other firms choose to invest; adopting a new technology may not be the best decision if others in the production chain will keep working with an old technology.

A variety of models that capture these economic problems give rise to multiple equilibria. In a range of parameters, different outcomes can arise in equilibrium depending on what agents expect others will do. For example, in models of bank runs, for an intermediate range of fundamentals, there is an equilibrium where agents attempt to withdraw their deposits and banks go bankrupt and another one where runs do not occur; in models of network externalities, equilibria predicting the prevalence of different networks coexist; in macroeconomic models, economic activity might depend on arbitrary shifts on expectations.

One important question left unanswered by models with multiple equilibria is what determines which equilibrium will be played. Will there be a bank run or not? Will people coordinate on Facebook, Orkut or Google+? Will economic activity recover next year?

This survey focuses on a stream of the literature that looks at the problem from a dynamic point of view. In the model of Frankel and Pauzner (2000), the seminal contribution to this literature, agents choose between two states (say, low and high) and get opportunities to revise their behavior according to a Poisson clock. Their instantaneous utility gain from being in the high state increases on a exogenous fundamental variable and on the fraction of agents in the high state. The fundamental moves according to a Brownian motion. ${ }^{1}$

As an illustration, agents might be on Facebook or not. The utility from being on Facebook depends on how good Facebook is (the exogenous fundamental) and how many people are on Facebook. At some random points in time, agents decide whether they want to be on Facebook or not. Here, the Poisson clock can be seen as an attention friction modeled in a reduced-form way. In other applications, the Poisson clock could be related to the maturity of a bond or an investment, or to the obsolescence of a machine.

\footnotetext{
${ }^{1}$ See also Burdzy, Frankel and Pauzner (2001).
} 
Section 2 presents the framework proposed by Frankel and Pauzner (2000) and some results for the general model. First, it presents results on equilibrium multiplicity and equilibrium uniqueness. Then, it shows a method to solve the social planner problem. Last, it applies mathematical results about bifurcation probabilities from Burdzy, Frankel and Pauzner (1998) to derive expressions for the equilibrium threshold in the limiting cases of vanishing shocks and vanishing frictions.

With this toolkit in hand, Section 3 shows analytical results for a case with linear preferences and presents applications of the basic framework to a variety of settings, such as network externalities, statistical discrimination and stimulus policies in macroeconomics. Besides generating insights for specific questions, the applications illustrate the potential of the model to accommodate a large set of economic problems. Last, Section 4 discusses extensions of the framework that allow for endogenous hazard rates, preemption motives and ex-ante heterogeneous agents.

Some of the material in this survey is not in the existing literature. Examples include: the general formulation for the social planner problem (Section 2.3); the model with asymmetric network externalities (Section 3.1.4); the study of efficiency in the model of statistical discrimination (Section 3.2.2); and the numerical solution for the macroeconomic model (Section 3.3.4).

Related strands of the literature. Among the alternative ways to understand agents' behavior in settings with strategic complementarities, the so-called global games literature is a particularly popular one (See Carlsson and Van Damme (1993), Morris and Shin (1998) and Morris and Shin (2003)). Models in this literature assume that agents have access to noisy private information about the economy. This removes the assumptions that all information is common knowledge and agents know what others are doing in equilibrium. One important result of this literature is that this can remove multiplicity of equilibria and lead to a unique outcome. Building on the theory of global games, a large literature has evolved. Angeletos and Lian (2016) offer a comprehensive survey of this literature.

Another strand of literature addresses this problem by incorporating dynamic and evolutionary processes. Examples include Matsui and Matsuyama (1995), Kandori, Mailath and Rob (1993) and Fudenberg and Harris (1992)). In simple 2x2 games, these different strands of the literature lead to similar predictions, but this is not true more generally (see Kim (1996) for a discussion). 


\section{The framework}

Time is continuous and runs forever. There is a continuum of agents with unit mass indexed by $i$. There are two possible actions $a_{i} \in\{0,1\}$.

Payoffs. An agent's payoff depends on her own action, the action of others and on some exogenous fundamental $\theta_{t}$. The action of other players is summarized by the proportion of players in action 1 , denoted by $n_{t}$. The instantaneous payoff of action 0 is given by a function $u_{0}\left(\theta_{t}, n_{t}\right)$ and the payoff of action 1 is written as $u_{1}\left(\theta_{t}, n_{t}\right)$, both continuously differentiable. We define the instantaneous relative gain of action 1 as

$$
\Delta u\left(n_{t}, \theta_{t}\right)=u_{1}\left(\theta_{t}, n_{t}\right)-u_{0}\left(\theta_{t}, n_{t}\right)
$$

Throughout these notes, we assume that $\Delta u(\cdot)$ is increasing in both arguments. It means that agents' incentives to choose action 1 are increasing on the exogenous fundamental and on the action of others (there are strategic complementarities).

Timing frictions. Agents cannot choose their actions at every period. They receive opportunities to revise their actions according to a Poisson clock (independent across agents) with arrival rate $\delta$. Once they choose an action they are locked in the chosen action until the Poisson shock hits again.

Simple examples. In a model where agents choose whether to participate in a given network or not, action 1 corresponds to taking part in the network and $\theta$ is a variable summarizing the intrinsic quality of the network. Thus $u_{1}\left(\theta_{t}, n_{t}\right)$ is the utility from participating, increasing in the quality of the network and in the number of people taking part, and $u_{0}\left(\theta_{t}, n_{t}\right)$ can be normalized to 0 . Alternatively, actions 0 and 1 can refer to two competing networks, agents choose between them. In this case, $u_{1}\left(\theta_{t}, n_{t}\right)$ is increasing in $n_{t}$ and $u_{0}\left(\theta_{t}, n_{t}\right)$ is decreasing in $n_{t}$. In a model of occupational choice along the lines of Matsuyama (1991), action 1 corresponds to an occupation that benefits from others choosing it as well (in the example, industry) and action 0 corresponds to an occupation with no such externalities (in the example, agriculture). In these examples, the timing frictions can be thought of as attention frictions.

The discounted expected gain of choosing 1 instead of zero at some date $\tau$ is given by

$$
V_{\tau}=\int_{\tau}^{\infty} e^{-(\rho+\delta)(t-\tau)} \mathbb{E}\left[\Delta u\left(\theta_{t}, n_{t}\right)\right] d t
$$


where $\rho>0$ is the discount rate of agents. Notice that an agent's decision today only affects her payoffs until she gets selected again. That is why the probability of not getting selected from $\tau$ to $t$, which is given by $e^{-\delta(t-\tau)}$, shows up in the expression above. Hence agents effectively discount the future at rate $\rho+\delta$. They choose 1 if $V_{\tau}>0$ and 0 if $V_{\tau}<0$.

An action is dominant if it is optimal for an agent to choose that action regardless of his beliefs about the action of others. Throughout this notes, we assume that the assumption below holds.

Assumption 1 (Existence of dominance regions). There exists $\tilde{\theta}$ and $\underset{\sim}{\theta}$ such that: if $\theta_{t}>\tilde{\theta}$ choosing 1 is a strictly dominant action; if $\theta_{t}<\tilde{\theta}$ choosing 0 is a strictly dominant action.

\subsection{Multiple equilibrium benchmark}

Here we analyze the case where the fundamental is constant and deterministic, i.e., $\theta_{t}=\theta$.

Take an agent deciding whether or not to choose action 1 when $n_{t}=n_{\tau}$. Suppose it is optimal for her to choose action 1 if she believes everyone that will choose after her will do the same. Then, players choosing after her have even an higher incentive to choose action 1 (because at time $\tau>t, n_{\tau}$ will be larger than $n_{t}$ ). Therefore, everyone choosing action 1 forever is an equilibrium. Conversely, if it is optimal for an agent to choose 0 if she believes everyone will do the same, then everyone choosing action 0 forever is an equilibrium.

To find the values of $\theta$ for which everyone choosing action 1 is an equilibrium, we compute two boundaries: (i) the boundary where an agent is indifferent between 0 and 1 if she believes that everyone will choose 1 in the future; (ii) the boundary where an agent is indifferent if she believes no one will choose action 1 in the future. Those are given by the solution of

$$
\int_{\tau}^{\infty} e^{-(\rho+\delta)(t-\tau)} \Delta u\left(\theta, n_{t}^{\uparrow}\right) d t=0
$$

and

$$
\int_{\tau}^{\infty} e^{-(\rho+\delta)(t-\tau)} \Delta u\left(\theta, n_{t}^{\downarrow}\right) d t=0
$$

where $n_{t}^{\uparrow}=1-\left(1-n_{\tau}\right) e^{-\delta(t-\tau)}$ and $n_{t}^{\downarrow}=n_{\tau} e^{-\delta(t-\tau)}$.

For any $\tau>t, n_{t}^{\uparrow}>n_{t}^{\downarrow}$. Hence, for a given $\theta$ and $n_{\tau}$, the LHS in (2) is larger than the LHS in (3). Therefore, for a given $n_{\tau}$, the value of $\theta$ that satisfies (2) must be smaller than the value of $\theta$ that satisfies (3). Intuitively, if agents expect all others to choose to action 1 , they are willing to do the same for lower values of $\theta$. 
Moreover, since $n_{t}^{\uparrow}$ is increasing in $n_{\tau}$ and $\Delta u\left(\theta, n_{t}^{\uparrow}\right)$ is increasing in both arguments, the value of $\theta$ that satisfies (2) is decreasing in $n_{\tau}$. Likewise, since $n_{t}^{\downarrow}$ is increasing in $n_{\tau}$, the value of $\theta$ that satisfies (3) is also decreasing in $n_{\tau}$.

The solutions of (2) and (3) are represented by the curves $\underline{\theta}$ and $\bar{\theta}$, respectively, in Figure 1. To the right of $\bar{\theta}$ choosing 1 is a dominant action; to the left of $\underline{\theta}$ choosing 0 is the dominant action; between the boundaries $\bar{\theta}$ and $\underline{\theta}$ we have multiple equilibria and agents' choices depend on their expectations about the actions of others.

Figure 1: Multiple equilibria with no shocks

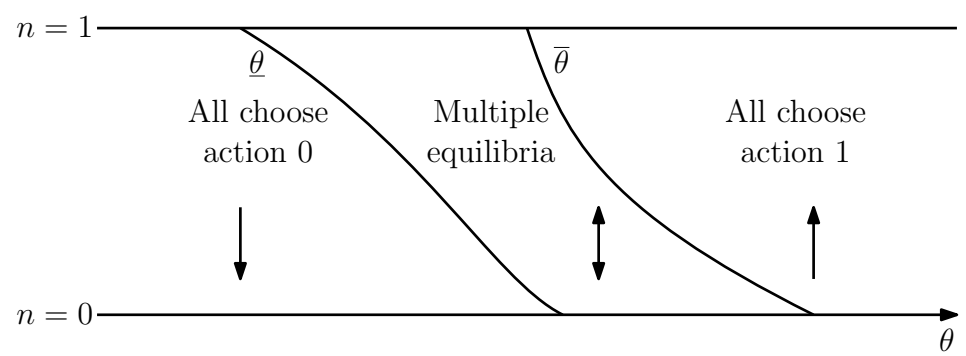

In this setting, agents can perfectly anticipate the future actions of others. Hence there is no strategic uncertainty. As we see next, this has important implications.

\subsection{Equilibrium uniqueness}

Now we assume that the fundamentals are subject to stochastic shocks. The key difference from the case without shocks is that now agents may not be able to perfectly anticipate the action of others in the future. Assume that

$$
d \theta_{t}=\mu d t+\sigma d Z_{t}
$$

where $d Z_{t}$ is a standard Brownian motion, $\mu$ is a constant drift and $\sigma>0$ is the volatility. The process above have two properties that are convenient. First, shocks are frequent: for any interval of length $d t>0$ we have that $\operatorname{Prob}\left(\theta_{t}=\theta_{t+d t}\right)=0$. Second, their increments are independent of history, i.e., $\theta_{t+d t}-\theta_{t}$ is independent of $\left(\theta_{s}\right)_{s \leq t}$. With shocks, multiplicity disappears.

Theorem 1 (Frankel and Pauzner, 2000). In the model with shocks, there is a unique equilibrium. Agents choose 1 when $\theta_{t}>\theta^{*}\left(n_{t}\right)$ and 0 when $\theta_{t}<\theta^{*}\left(n_{t}\right)$, where $\theta^{*}(\cdot)$ is a decreasing function.

Figure 2 illustrates the result. An agent choosing an action at some point on the equilibrium threshold must be indifferent between both actions. Formally, for every $n_{\tau} \in$ 
Figure 2: Unique equilibrium with shocks

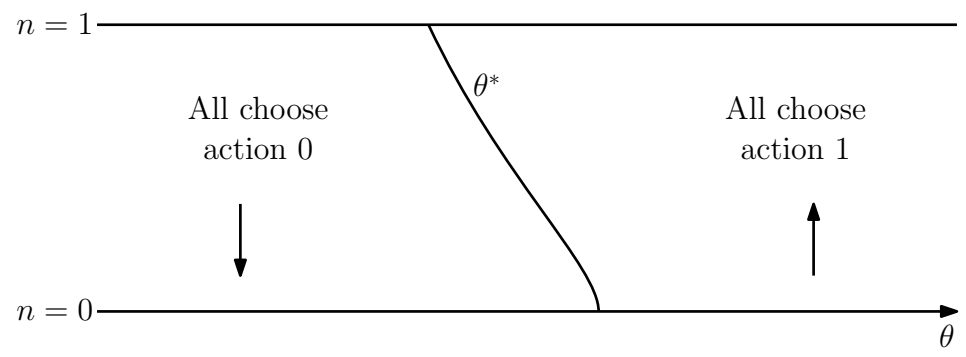

$[0,1], \theta^{*}\left(n_{\tau}\right)$ must solve:

$$
\int_{\tau}^{\infty} e^{-(\rho+\delta)(t-\tau)} \mathbb{E}\left[\Delta u\left(\theta_{t}, n_{t}\right) \mid \theta^{*}, \theta^{*}\left(n_{\tau}\right), n_{\tau}\right] d t=0
$$

where the operator $\mathbb{E}\left[\cdot \mid \tilde{\theta}, \theta_{\tau}, n_{\tau}\right]$ denotes agents' expectation when the current state is $\left(\theta_{\tau}, n_{\tau}\right)$ and they expects others to play according to the threshold $\tilde{\theta}$.

To understand the intuition behind the result, it is useful to go through the proof. The proof here is slightly different from the original proof in Frankel and Pauzner (2000) and a bit more similar to the proof of equilibrium uniqueness in Frankel, Morris and Pauzner (2003), even though that is a static global-game model.

\subsubsection{Proof of equilibrium uniqueness}

A key assumption is that we have dominance regions, as represented by the boundaries $\underline{\theta}_{0}$ and $\bar{\theta}_{0}$ in Figure 3. Instead of looking for a strategy profile that is a Nash equilibrium, we are going to look for strategy profiles that survive the iterated elimination of strictly dominated strategies (hereafter, IESDS). This is a less restrictive equilibrium concept, since every Nash Equilibrium also survives IESDS (but not all strategy that survive IESDS is a Nash Equilibrium).

A strategy here is a map from every possible history to a probability of choosing action 1 (i.e., a map that prescribes what an agent selected by the Poisson process will choose in every contingency).

Iterations from the left. Let's start a process of elimination of strictly dominated strategies. Notice that any strategy that prescribes playing 1 to the left of $\underline{\theta_{0}}$ is strictly dominated. Therefore, we can remove those strategies from our game (we can also remove strategies that prescribe playing 0 to the right of $\bar{\theta}_{0}$, but let's leave them there for a while).

We can now ask the same question we asked to construct the boundary $\underline{\theta}_{0}$ : when is an agent indifferent between actions 0 and 1 under the most optimistic belief about 
Figure 3: Dominance regions

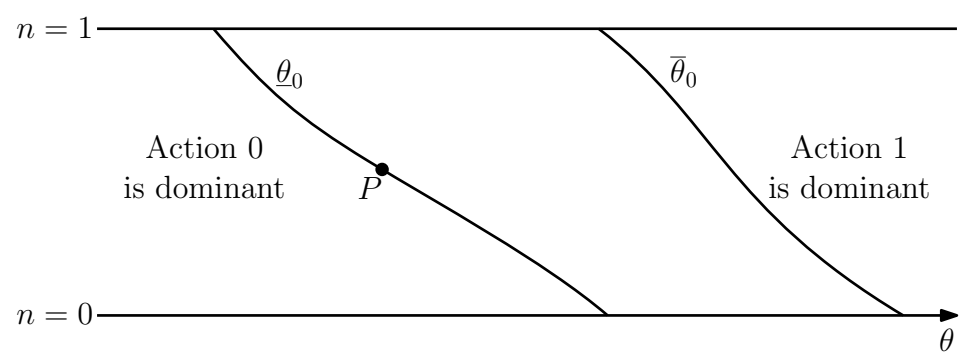

$\left(n_{t}\right)_{t \geq \tau}$ ? The difference now is that, after eliminating the strictly dominated strategies, the meaning of 'most optimistic belief' changed. It is no longer possible to assume that agents will play action 1 always, since we eliminated strategies that prescribed playing 1 to the left of $\underline{\theta}_{0}$ and the probability of $\theta_{t}$ crossing the boundary $\underline{\theta}_{0}$ at some point is positive. Thus, the most optimistic belief now is the one in which other agents play 1 in any state $\left(\theta_{t}, n_{t}\right)$, except in those states to the left $\underline{\theta}_{0}$. Under those beliefs, an agent choosing at the point $P$ in Figure 3 will no longer be indifferent between action 1 and 0 . She will strictly prefer to choose action 0 (if she was indifferent under a more optimistic belief, now she is strictly preferring to choose action 0 ). Thus, the boundary where an agent is indifferent under the most optimistic belief shifts to the right, as illustrated by the boundary $\underline{\theta}_{1}$ in Figure 4 .

Figure 4: First round of elimination of dominated strategies

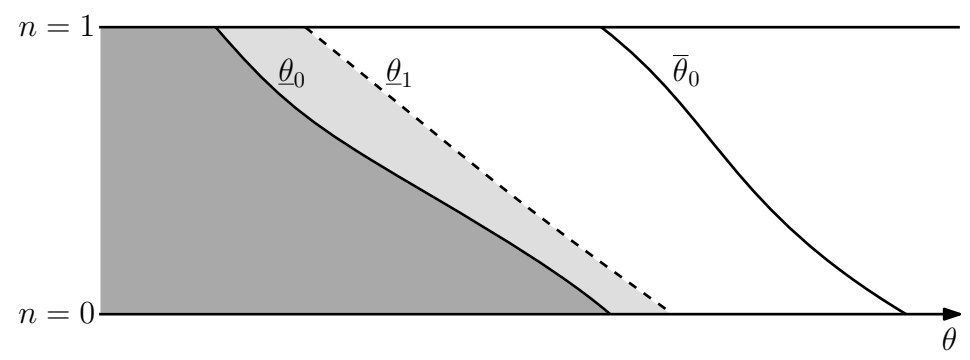

Now we can move to the second round of elimination of strictly dominated strategies. After eliminating the strategies that imply playing 1 in the dark gray area in Figure 4, the strategies that imply playing 1 in the light gray area also become strictly dominated strategies (agents wouldn’t choose it even under the most optimistic belief possible). Thus, we further eliminate the strategies that imply playing 1 to the left of $\underline{\theta}_{1}$ and we get a boundary $\underline{\theta}_{2}$. Continuing this process indefinitely we get a boundary $\underline{\theta}_{\infty}$, as illustrated in Figure 5. Notice that $\underline{\theta}_{\infty}$ must be to the left of $\bar{\theta}_{0}$, since in the latter agents believe everyone will always choose 0 , while in the former they believe that everyone to the left 
of $\underline{\theta}_{\infty}$ will choose 0 (and thus they require a lower initial $\theta$ to be indifferent).

Figure 5: Convergence of iterations from the left

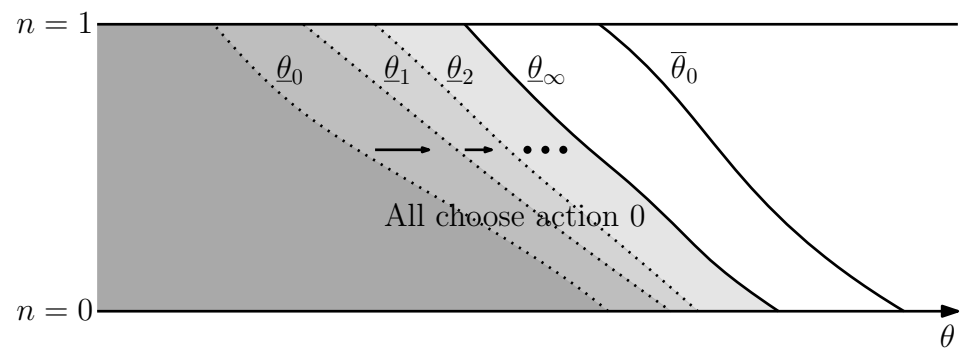

So far we know that in any strategy that survives the iterated elimination of strictly dominated strategies agents play 0 to the left of $\underline{\theta}_{\infty}$. Notice also that an agent making a decision on the threshold $\underline{\theta}_{\infty}$ must be indifferent between 0 and 1 (otherwise the iterations could continue). Thus, everyone playing 1 to the right of $\underline{\theta}_{\infty}$ and 0 to the left of it must be a Nash equilibrium. We have just proved equilibrium existence.

Iterations from the right. We can repeat an analogous procedure starting from the boundary $\bar{\theta}_{0}$, by first eliminating the strategies in which agents play 0 to the right of $\bar{\theta}_{0}$. The only difference is that now we will always consider the most pessimistic beliefs possible about $n_{t}$ in each iteration, given the strategies that we have already eliminated. This process will yield a boundary $\bar{\theta}_{\infty}$. In any equilibrium that survives the iterated elimination of strictly dominated strategies, agents must play 0 to the left of $\bar{\theta}_{\infty}$. As for $\underline{\theta}_{\infty}$, notice that everyone playing according to $\bar{\theta}_{\infty}$ is an equilibrium, since agents are indifferent between both actions on the threshold $\bar{\theta}_{\infty}$ if they believe others will play according to this threshold.

Limit of iterations coincide. Notice that $\bar{\theta}_{\infty}$ cannot be to the left of $\underline{\theta}_{\infty}$, otherwise there would be no strategy that survives IESDS (we know it cannot be true since a Nash Equilibrium exists and every Nash equilibrium survives IESDS). ${ }^{2}$

If $\bar{\theta}_{\infty}$ and $\underline{\theta}_{\infty}$ coincide for every $n$, then we have an essentially unique equilibrium. ${ }^{3}$ Suppose by contradiction that it is not the case, as exemplified in Figure 6. Then, we can always get a translation of $\bar{\theta}_{\infty}$ that lies entirely to the left of $\underline{\theta}_{\infty}$, but touches it in at least one point, as represented by the curve $\bar{\theta}_{\infty}^{\prime}$ in the figure.

\footnotetext{
${ }^{2}$ If $\bar{\theta}_{\infty}$ is to the left of $\underline{\theta}_{\infty}$, then there is a subset $V$ of the state space such that: (i) every strategy that survives IESDS requires playing 1 when $\left(\theta_{t}, n_{t}\right) \in V$; (ii) every strategy that survives IESDS requires playing 0 when $\left(\theta_{t}, n_{t}\right) \in V$. Obviously, no strategy can satisfy (i) and (ii) at the same time.

${ }^{3}$ The word "essentially" appears because agents can do whatever they want on the threshold, but those states have zero mass.
} 
Figure 6: Translations

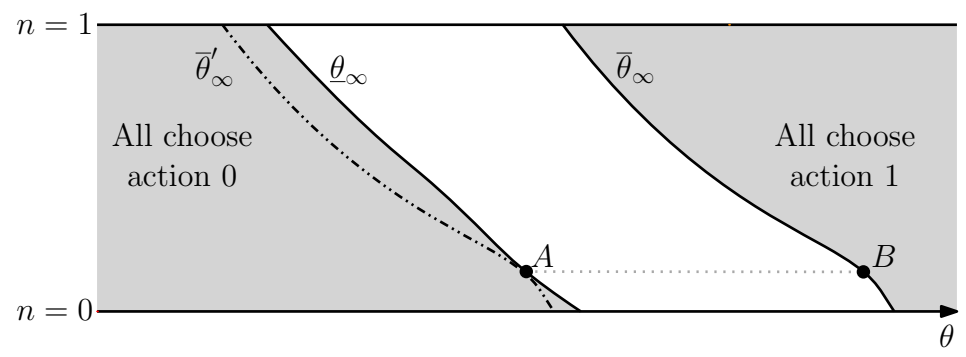

Consider a player that is choosing at point $A$ and believes everyone will play according to $\bar{\theta}_{\infty}^{\prime}$. Her payoff of choosing 1 must be strictly positive, since it would be zero if she believed everyone would play according to $\underline{\theta}_{\infty}$ and therefore choose action 1 less often for any Brownian path (remember that $\underline{\theta}_{\infty}$ is an equilibrium).

Consider now the payoff of player $B$. For player $B$, the increments $\left(\theta_{\tau+s}-\theta_{\tau}\right)$ follow the same distribution as the increments for player $A$, since $\bar{\theta}_{\infty}^{\prime}$ is a translation of $\bar{\theta}_{\infty}$. In other words, for any Brownian path $\left(Z_{t}\right)_{t \geq \tau}$ player $B$ observes the same fundamentals as player $A$ plus a positive constant (that is the distance between their thresholds). Thus, for any Brownian path, player $A$ will have $\theta_{t}$ to the left (right) of her threshold if, and only if, player $B$ also has her $\theta_{t}$ to the left (right) of her threshold. Thus, they will always experience the same dynamics of $n_{t}$, but player $B$ always experiences a higher fundamental. Thus, the relative payoff of player $B$ of choosing action 1 (which we know to be zero, since $\bar{\theta}_{\infty}$ is an equilibrium) must be higher than that of player $A$. Thus we get the contradiction:

$$
0=(\text { Player } B \text { 's gain of choosing } 1)>(\text { Player } A \text { 's gain of choosing } 1)>0 \text {. }
$$

\subsubsection{Relation to 'global games'}

The basic global-game models are static coordination games, where agents observe a noisy idiosyncratic signal on the fundamental variable that determines payoffs $(\theta) .{ }^{4}$ Owing to this idiosyncratic noise, as long as there are dominance regions, it is never common knowledge that the fundamental variable lies in a region where the equilibrium could be driven

\footnotetext{
${ }^{4}$ There are several dynamic models employing the global-game methodology. Examples include Angeletos, Hellwig and Pavan (2007), Dasgupta (2007), Steiner (2008), Chassang (2010), Dasgupta, Steiner and Stewart (2012), Kováč and Steiner (2013) and Mathevet and Steiner (2013). However, dynamics is not needed to generate equilibrium uniqueness and the basic insights from the methodology. This section highlights the analogies between the dynamic framework considered here and static global-game models.
} 
by self-fulfilling beliefs. Moreover, agents do not know what others are doing in equilibrium. Based on their own signals, agents form expectations about others' information and expectations about others' expectations (higher-order expectations).

The intuition for equilibrium uniqueness in global games is similar to the intuition for Theorem 1. An agent with a signal about fundamentals very close to the region where, say, action 1 is dominant considers that others might think that fundamentals lie in the dominance region and will choose action 1 . Hence action 1 is the best choice for this agent. This triggers a similar process of iterated elimination of strictly dominated strategies that end up with a unique rationalizable strategy.

This dynamic framework seems different because all information is common knowledge. Morris (2014) shows that this intuition is misleading: what matters is not players' actual beliefs but what their beliefs were at their last opportunity to switch actions. Now consider that an event is effectively known by a player if she knew it the last time she had an opportunity to change behavior. Then there is a tight connection between the lack of effective common knowledge here and the lack of common knowledge in global-game models (See Morris (2014)).

\subsection{The social planner's problem}

We now study the social planner's problem. Let's say welfare is given by the discounted sum of individual agents' payoffs (which depend on others' actions as well). Thus, the planner maximizes:

$$
\mathbb{E}\left[\int_{t=\tau}^{\infty} e^{-\rho(t-\tau)} W\left(\theta_{t}, n_{t}\right) d t\right]
$$

where

$$
W(\theta, n)=n u_{1}(\theta, n)+(1-n) u_{0}(\theta, n) .
$$

At each date $t$, the planner chooses the proportion $\phi_{t} \in[0,1]$ of agents that received a chance to switch actions that will pick action 1 .

Suppose that at a given date $\tau$ it is optimal for the planner to choose $\phi_{\tau}<1$ and consider the following deviation: the planner increases $\phi_{\tau}$ in $\Delta \phi>0$ units today, but keeps the future values of $\phi_{t}$ unchanged, for any realization of the Brownian path. Increasing $\phi_{t}$ by $\Delta \phi$ today, raises $n_{\tau}$ by $\delta \Delta \phi d t \equiv d \phi$. But at a given date $t>\tau$ a proportion $e^{-\delta(t-\tau)}$ has already been selected again. Therefore, the resulting change in $n$ at time $t \geq \tau$ is:

$$
d n_{t}=d \phi e^{-\delta(t-\tau)} .
$$


This deviation is not profitable if

$$
\int_{\tau}^{\infty} \mathbb{E}_{\tau}\left[e^{-\rho(t-\tau)} \frac{\partial W\left(\theta_{t}, n_{t}\right)}{\partial n} e^{-\delta(t-\tau)} d \phi\right] d t \leq 0
$$

which becomes

$$
\int_{\tau}^{\infty} e^{-(\rho+\delta)(t-\tau)} \mathbb{E}_{\tau}\left[\frac{\partial W\left(\theta_{t}, n_{t}\right)}{\partial n}\right] d t \leq 0
$$

Now assume that $\phi_{\tau}>0$ and the planner chooses a similar deviation, but with $d \phi<0$. The same reasoning implies that this deviation is not profitable if

$$
\int_{\tau}^{\infty} e^{-(\rho+\delta)(t-\tau)} \mathbb{E}_{\tau}\left[\frac{\partial W\left(\theta_{t}, n_{t}\right)}{\partial n}\right] d t \geq 0
$$

This implies the following necessary conditions for optimality: if $\phi_{\tau}=0$ then (7) holds; if $\phi_{\tau}=1$ then (8) holds; if $\phi_{\tau} \in(0,1)$ then (7) holds with equality. But those are exactly the necessary (and sufficient) conditions for a Nash Equilibrium in the game where the relative payoff $\Delta u(\theta, n)$ is replaced by $\frac{\partial W(\theta, n)}{\partial n}$ (see equation (1)).

Hence, if we find the set of Nash Equilibria in this modified game, we have found all the candidates for the planner solution. But as long as $\frac{\partial W(\theta, n)}{\partial n}$ satisfies the same conditions we imposed on $\Delta u(\theta, n)$ and we have shocks, the equilibrium is unique and therefore these necessary conditions are also sufficient for optimality.

Therefore the planner plays a modified game, in which the flow gain of choosing 1 is given by

$$
\frac{d W(\theta, n)}{d n}=\underbrace{\left[u_{1}(\theta, n)-u_{0}(\theta, n)\right]}_{\Delta u(\theta, n)}+\underbrace{\left[n \frac{\partial u_{1}(\theta, n)}{\partial n}+(1-n) \frac{\partial u_{0}(\theta, n)}{\partial n}\right]}_{\text {Externality }} .
$$

The agent considers the first term in brackets, but does not consider the externality on others. The difference between the planner's problem and the agents' problem is the second term in brackets. Looking at how they relate, we might get some intuition for how the planner's threshold relates to the agents' threshold.

\subsection{Bifurcation probabilities}

We now discuss other results that will be useful when explicitly caracterizing the equilibrium in some particular cases. For simplicity of the exposition, assume $\mu=0$ hereafter, unless stated otherwise. Suppose agents play according to some decreasing threshold $\theta^{*}(n)$ (as we have seen, they do that in equilibrium). ${ }^{5}$ Let's look to the limiting case

\footnotetext{
${ }^{5}$ There are additional technical requirements that are always satisfied by an equilibrium threshold. In particular, $\theta^{*}(\cdot)$ must be a Lipschitz function.
} 
where shocks are very small, i.e., $\sigma \rightarrow 0 .{ }^{6}$ What is the dynamics of $n_{t}$ when the economy starts off at some point on the threshold $\theta^{*}(n)$ ?

This mathematical problem is studied by Burdzy, Frankel and Pauzner (1998). They show that, as $\sigma \rightarrow 0$, the economy either bifurcates in the direction of $n=0$ or in the direction of $n=1$, and never comes back. Moreover, the time it takes for the economy to start heading off in one direction or the other goes to zero. The economy instantaneously moves in one of the directions indicated by the arrows in Figure 7.

Figure 7: Bifurcation probabilities

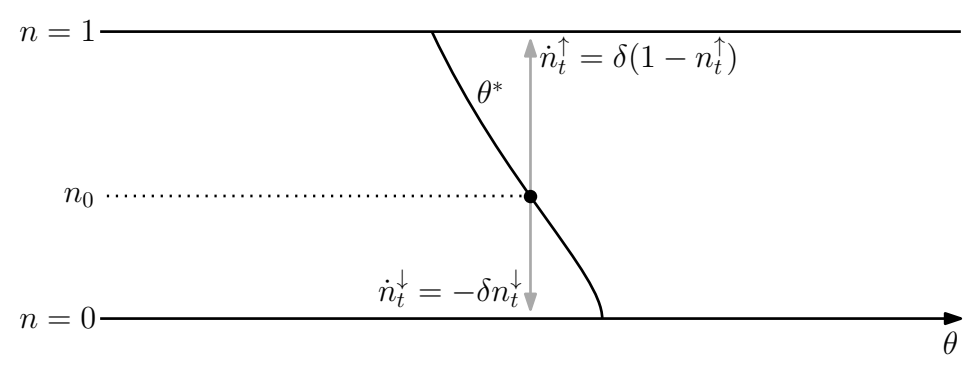

When the economy is at the threshold, a small positive shock pushes the economy to the right of it and $n_{t}$ goes up. Negative shocks push the economy to the left of the threshold and thus $n$ goes down. Since the threshold is negatively sloped, once the economy stays away for a while to the right of the threshold, as $n$ goes up, it drifts further away from the threshold and never comes back. The same logic applies when a sequence of negative shocks hit. Since shocks are very small and frequent, the bifurcation happens very quickly. Burdzy, Frankel and Pauzner (1998) shows that as $\sigma \rightarrow 0$, the time until it bifurcates converges to zero.

But what is the probability of the economy moving in either direction? Burdzy, Frankel and Pauzner (1998) show that it is proportional to the speed that the economy moves in either direction. More specifically:

$$
\frac{\text { Prob(bifurcate up) }}{\text { Prob(bifurcate down) }}=\frac{\text { initial speed of } n_{t} \text { if bifurcates up }}{\text { initial speed of } n_{t} \text { if bifurcates down }}=\frac{\left|\dot{n}_{0}^{\uparrow}\right|}{\left|\dot{n}_{0}^{\downarrow}\right|}
$$

which implies that

$$
\operatorname{Prob}(\text { bifurcate up })=1-n_{0} \text { and } \operatorname{Prob}(\text { bifurcate down })=n_{0} .
$$

To understand the intuition, suppose $\sigma \approx 0$ and that $n_{t}$ moves up very quickly when to the right of the threshold and goes down very slowly when to the left of it. Then, a small

\footnotetext{
${ }^{6}$ The result presented in this section holds for $\mu, \sigma \rightarrow 0$, regardless of the relative speed at which $\mu$ and $\sigma$ go to zero.
} 
sequence positive shocks moves the economy very far from the threshold, but we need a very large sequence of negative shocks to get far away from it. If positive and negative shocks are equally likely, it makes sense to think that it is more likely that economy will bifurcate up.

\subsection{Results for limiting cases}

Although in general it is not easy to compute the equilibrium threshold $\theta^{*}(n)$, in two limiting cases the model is especially tractable: (i) when $\sigma \rightarrow 0$ and (ii) when $\delta \rightarrow \infty$. Besides providing approximate results for the cases with small shocks and small frictions, these limiting cases are interesting for helping us to understand how small departures from the case with no shocks and/or no timing frictions affect equilibrium outcomes.

\subsubsection{Vanishing shocks}

When $\sigma \rightarrow 0$, we can use the results from Burdzy, Frankel and Pauzner (1998), namely that the time until bifurcation converges to zero and the bifurcation probabilities in (11), to compute the beliefs of an agent deciding on the threshold. The indifference condition that solves for the equilibrium threshold in (4) becomes:

$$
\left(1-n_{0}\right) \int_{0}^{\infty} e^{-(\rho+\delta) t} \Delta u\left(\theta^{*}\left(n_{0}\right), n_{t}^{\uparrow}\right) d t+n_{0} \int_{0}^{\infty} e^{-(\rho+\delta) t} \Delta u\left(\theta^{*}\left(n_{0}\right), n_{t}^{\downarrow}\right) d t=0,
$$

where $n_{t}^{\uparrow}=1-\left(1-n_{0}\right) e^{-\delta t}$ and $n_{t}^{\downarrow}=n_{0} e^{-\delta t}$. Solving the expression for $\theta^{*}$ we get the equilibrium threshold. Alternatively, we can apply the change of variables $v=n_{t}^{\uparrow}$ and $v=n_{t}^{\downarrow}$ in the integrals above to get:

$$
\int_{0}^{n_{0}}\left(\frac{v}{n_{0}}\right)^{\frac{\rho}{\delta}} \frac{1}{\delta} \Delta u\left(\theta^{*}\left(n_{0}\right), v\right) d v+\int_{n_{0}}^{1}\left(\frac{1-v}{1-n_{0}}\right)^{\frac{\rho}{\delta}} \frac{1}{\delta} \Delta u\left(\theta^{*}\left(n_{0}\right), v\right) d v=0 .
$$

Figure 8 depicts the equilibrium. The curves $\theta^{\text {opt }}$ and $\theta^{\text {pes }}$ represent the two extreme equilibria of the game with no shocks - ('opt' and 'pes' stand for optimistic and pessimistic beliefs, respectively). Notice that the threshold $\theta^{*}$ touches $\theta^{\text {opt }}$ when $n=0$, since the bifurcation probabilities imply that agents expect $n_{t}$ to go up forever with probability one at the state $\left(\theta^{*}(0), 0\right)$. Similarly, $\theta^{*}$ touches $\theta^{\text {pes }}$ when $n=1$, since the system bifurcates down with probability one at the state $\left(\theta^{*}(1), 1\right)$.

\subsubsection{Vanishing frictions}

With vanishing frictions $(\delta \rightarrow \infty)$ agents are allowed to switch actions at almost every period. To derive the equilibrium in this case, it is useful to write down our environment 
Figure 8: Equilibrium with vanishing shocks

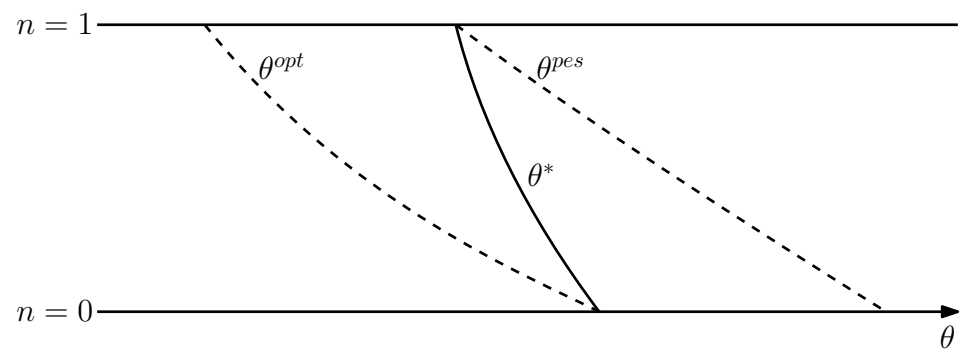

in a different unit of time. Suppose that date $t$ in the new unit of time is represented by $\tilde{t}=\delta t$. For instance, if we were initially measuring time in years and $\delta=12$, it means that we are now measuring time in months. The arrival rate of the Poisson process becomes $\tilde{\delta}=1$; the discount rate becomes $\tilde{\rho}=\rho / \delta$; the variance of the change in fundamentals between $\tilde{t}$ and $\tilde{t}+1$ becomes $\tilde{\sigma}^{2}=\sigma^{2} / \delta .^{7}$ Sending $\delta$ to infinity is thus equivalent to taking the limit when $\rho \rightarrow 0$ and $\sigma \rightarrow 0$.

We know from the previous section that when $\sigma \rightarrow 0$, the threshold is characterized by (13). Taking the limit when $\rho \rightarrow 0$ in (13), we get that the threshold $\theta^{*}\left(n_{0}\right)$ is given by: ${ }^{8}$

$$
\int_{0}^{1} \Delta u\left(\theta^{*}\left(n_{0}\right), v\right) d v=0 .
$$

Notice that the expression above does not depend on $n_{0}$, and thus $\theta^{*}\left(n_{0}\right)$ is constant. Figure 9 shows the equilibrium in this case.

Figure 9: Equilibrium with vanishing frictions

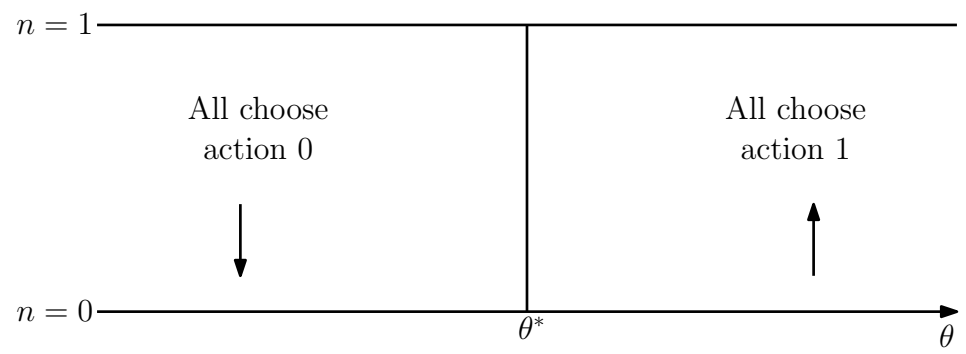

The equilibrium does not depend on the current value of $n$, just on the fundamental. The result may seem to follow immediately from the fact that the $n_{t}$ moves very quickly in this economy (true), which would imply that the current value of $n_{t}$ is irrelevant (false). This intuition is incorrect because although the economy can move very quickly from a

\footnotetext{
${ }^{7}\left(\theta_{t+s}-\theta_{t}\right) \sim N\left(0, \sigma^{2} s\right)$, for every $t$ and $s$. Thus, $\sigma^{2}$ represents the variance of $\left(\theta_{t+1}-\theta_{t}\right)$.

${ }^{8}$ This expression coincides with the equilibrium condition in a static global game where agents have a diffuse prior, get a noisy signal about $\theta$ and their payoffs are $\Delta u(\theta, n)$. See Morris and Shin (2003).
} 
low $n$ to a high $n$, the next opportunity to change behavior also comes very quickly, so the agent effectively discounts the future at rate $\rho+\delta$.

The intuition for the irrelevance of the current value of $n$ is the following. An agent starting at $n=0$ will experience $n$ from 0 to $h^{\dagger}$, where $h^{\dagger}$ is uniformly distributed in $[0,1]$; while an agent starting at $h=1$ will experience $h$ from 1 to $1-h^{\dagger}$. Hence the agent starting at $n=0$ will experience a lower range of values of $n$ than the agent starting at $n=1$ (before their next opportunity to choose again). However, for the agent starting at $n=0$, the economy moves up very quickly at lower values of $n$, but very slowly as $n$ approaches 1 , so the last agents to change their decision will spend relatively more time at high values of $n$. As it turns out, this effects exactly offset the previous one, so agents at $n=0$ and $n=1$ are indifferent between actions 0 and 1 for the same value of $\theta .^{9}$

\subsection{The planner's problem with vanishing shocks}

For the planner, it makes no difference whether there are no shocks or shocks are very small (the planner does not face a coordination problem with itself). Hence the model can be solved in two different ways: assuming vanishing shocks $(\sigma \rightarrow 0)$ or no shocks $(\sigma=0)$.

The case $\sigma \rightarrow 0$. As shown in section 2.3, as long as $\partial W(\theta, n) / \partial n$ satisfies the same conditions we imposed on $\Delta u(\theta, n)$ and we have shocks, the planner's problem can be seen as analogous to the agents' problem, only with different payoffs. Considering vanishing shocks, we can then apply the bifurcation probabilities to solve for the planner's threshold. The indifference condition characterizing the planner's threshold is given by

$$
\left(1-n_{0}\right) \int_{0}^{\infty} e^{-(\rho+\delta) t} \frac{\partial W\left(\theta^{P}, n_{t}^{\uparrow}\right)}{\partial n} d t+n_{0} \int_{0}^{\infty} e^{-(\rho+\delta) t} \frac{\partial W\left(\theta^{P}, n_{t}^{\downarrow}\right)}{\partial n} d t=0,
$$

where again $n_{t}^{\uparrow}=1-\left(1-n_{0}\right) e^{-\delta t}$ and $n_{t}^{\downarrow}=n_{0} e^{-\delta t}$, and $\partial W(\theta, n) / \partial n$ is given by equation (9). Solving this equation for $\theta^{P}$ gives us the planner's threshold as a function of $n_{0}$.

The case $\sigma=0$. We can also compute the planner's threshold by searching for the curve $\theta^{P}\left(n_{0}\right)$ along which the planner is indifferent between sending agents to network 1 forever, or to network 0 forever, assuming there are no shocks to fundamentals. Assuming $\sigma=0$, for a given $n_{0}$ the planner is indifferent between an upward or a downward path for $n_{t}$

\footnotetext{
${ }^{9}$ See also the explanation in Burdzy, Frankel and Pauzner (2001).
} 
when $\theta^{P}$ satisfies:

$$
\begin{aligned}
\int_{0}^{\infty} e^{-\rho t}\left[n_{t}^{\uparrow} u_{1}\left(\theta^{P}, n_{t}^{\uparrow}\right)+\left(1-n_{t}^{\uparrow}\right)\right. & \left.u_{0}\left(\theta^{P}, n_{t}^{\uparrow}\right)\right] d t \\
& =\int_{0}^{\infty} e^{-\rho t}\left[n_{t}^{\downarrow} u_{1}\left(\theta^{P}, n_{t}^{\downarrow}\right)+\left(1-n_{t}^{\downarrow}\right) u_{0}\left(\theta^{P}, n_{t}^{\downarrow}\right)\right] d t .
\end{aligned}
$$

The two approaches must lead to the same resulting threshold $\theta^{P}\left(n_{0}\right)$.

\section{Applications}

We now consider applications of the framework presented in Section 2 to a variety of settings. All these models can be seen as particular cases of the basic framework, but their implications are substantially different. In particular, the efficiency results are quite different across models.

\subsection{Linear utility}

We now consider a particular case of the model where the agents' utility is a linear function of $\theta_{t}$ and $n_{t}$, as in Guimaraes and Pereira (2016). This case is particularly tractable as it allows for closed form solutions in the limiting cases previously discussed.

Agents' utility functions are given by

$$
u_{t}^{0}\left(\theta_{t}^{0}, n_{t}\right)=\theta_{t}^{0}+\nu^{0}\left(1-n_{t}\right) \text { and } u_{t}^{1}\left(\theta_{t}^{1}, n_{t}\right)=\theta_{t}^{1}+\nu^{1} n_{t},
$$

where $\theta_{t}^{j}$ represents the fundamentals affecting the flow-payoff of action $j$ and follows a Brownian motion with drift $\mu_{j}$ and variance $\sigma_{j}^{2}$. The flow-utility of those choosing action $j$ increases linearly in the mass of agents taking the same action. We can write the relative payoff function as

$$
\Delta u\left(\theta_{t}, n_{t}\right)=\theta_{t}+\gamma n_{t}
$$

where $\theta_{t} \equiv \theta_{t}{ }^{1}-\theta_{t}{ }^{0}-\nu^{0}$ follows a Brownian motion with drift $\mu=\mu_{1}-\mu_{0}$ and variance $\sigma=\sigma_{0}^{2}+\sigma_{1}^{2}$, and $\gamma \equiv \nu^{0}+\nu^{1}$. Assume $\nu^{0}$ and $\nu^{1}$ are such that $\gamma>0$. Notice $\Delta u(\cdot)$ satisfies all the assumptions listed in section 2 .

This is a general linear case. When $\mu_{0}=\sigma_{0}^{2}=\nu^{0}=0$, one of the actions yields a constant payoff. When $\nu^{0}=\nu^{1}$, we have a model of network externalities that are the same for both networks. 


\subsubsection{Equilibrium threshold in limiting cases}

As in the general case, an agent who receives an opportunity to revise her choice at time $\tau$ will choose action $a_{i}=1$ if the discounted relative payoff of doing so is positive:

$$
\mathbb{E} \int_{\tau}^{\infty} e^{-(\rho+\delta)(t-\tau)}\left(\theta_{t}+\gamma n_{t}\right) d t>0 .
$$

If the inequality is reversed, the agent will choose $a_{i}=0$. Theorem 1 ensures there is a unique equilibrium in which agents play according to a downward sloping threshold. If we focus on the limiting case with $\mu, \sigma \rightarrow 0$, we can simply apply the bifurcation probabilities of section 2.4 to compute the equilibrium threshold. Substituting the expression for $\Delta u\left(\theta_{t}, n_{t}\right)$ in this tractable case into (12), we have that the indifference condition for agents is given by

$$
\left(1-n_{0}\right) \int_{0}^{\infty} e^{-(\rho+\delta) t}\left(\theta^{l}+\gamma n_{t}^{\uparrow}\right) d t+n_{0} \int_{0}^{\infty} e^{-(\rho+\delta) t}\left(\theta^{l}+\gamma n_{t}^{\downarrow}\right) d t=0 .
$$

Solving for $\theta^{l}$, we get an explicit expression for the linear equilibrium threshold:

$$
\theta^{l}\left(n_{0}\right)=-\frac{\gamma \delta}{\rho+2 \delta}-\frac{\gamma \rho}{\rho+2 \delta} n_{0}
$$

It is also possible to explicitly characterize the equilibrium threshold in the other tractable limiting case: when timing frictions vanish. It suffices to substitute $\Delta u\left(\tilde{\theta}^{l} n\right)=$ $\tilde{\theta}^{l}+\gamma n$ into (14) and solve for $\tilde{\theta}^{l}$. Analogously, one could simply take the limit of the right-hand side of (17) as $\delta \rightarrow \infty$ to find that the threshold is given by $\tilde{\theta}^{l}=-\gamma / 2$.

\subsubsection{The planner problem with network externalities}

We now use the model with linear utility to study efficiency in an environment with network externalities. The expression in (6) and simple algebra yield the planner's instantaneous payoff:

$$
W(\theta, n)=\theta^{0}+\nu^{0}+\left(\theta-\nu^{0}\right) n+\gamma n^{2}
$$

hence

$$
\frac{d W(\theta, n)}{d n}=\underbrace{[\theta+\gamma n]}_{\Delta u(\theta, n)}+\underbrace{\gamma n-\nu^{0}}_{\text {Externality }} .
$$

Notice that increasing $n$ implies a positive externality term if and only if $\gamma n>\nu^{0}$, i.e, if $\nu^{1} n>\nu^{0}(1-n)$. Intuitively, the choice of action 1 increases the payoff of agents locked in action 1 but reduces the payoff of those locked in action 0 . Therefore, the sign of the externality term depends on which of those effects dominate, which in turn depends on 
the amount of agents in each action and on the relative importance of the externality for them. From section 2.3, the planner recommends action 1 whenever

$$
\int_{0}^{\infty} e^{-(\rho+\delta) t}\left[\theta_{t}-\nu^{0}+2 \gamma n_{t}\right] d t>0 .
$$

This expression for the planner is quite similar to the condition for an agent in (16). The only substantial difference is that $\gamma$ is multiplied by 2 , indicating that the planner gives more importance to externalities.

Limiting case. Assume $\mu, \sigma \rightarrow 0$. From section 2.6, we know the indifference condition for the planner becomes

$$
\left(1-n_{0}\right) \int_{0}^{\infty} e^{-(\rho+\delta) t}\left[\theta^{P}-\nu^{0}+2 \gamma n_{t}^{\uparrow}\right] d t+n_{0} \int_{0}^{\infty} e^{-(\rho+\delta) t}\left[\theta^{P}-\nu^{0}+2 \gamma n_{t}^{\downarrow}\right] d t=0 .
$$

Solving for $\theta^{P}$, we find that the planner plays according to the threshold:

$$
\theta^{P}\left(n_{0}\right)=\nu^{0}-\frac{2 \gamma \delta}{\rho+2 \delta}-\frac{2 \gamma \rho}{\rho+2 \delta} n_{0} .
$$

\subsubsection{Symmetric network effects}

We now assume $\nu \equiv \nu^{0}=\nu^{1}$ so that externalities are the same for both networks. Hence increasing $n$ implies a positive externality term whenever $n>0.5$ and a negative term otherwise. Using $\nu^{0}=\gamma / 2$ in (18) and doing some algebra yields:

$$
\theta^{P}\left(n_{0}\right)=-\frac{\gamma \delta}{\rho+2 \delta}+\frac{\gamma \rho}{2(\rho+2 \delta)}-\frac{2 \gamma \rho}{\rho+2 \delta} n_{0} .
$$

Guimaraes and Pereira (2016) use this model to describe consumers' choices between two competing standards, such as the QWERTY keyboard and the alternative (and allegedly better) option, the Dvorak keyboard. The utility functions capture agents' preference for higher-quality products and for using the standard that is widespread. One can think that the QWERTY standard incidentally spread out before the higher-quality Dvorak standard became available. We have then a conflict between the two features of preferences: one product has the biggest network of consumers, but the other product is the highest-quality one. Is there room for interventions such as subsidies to eliminate inefficiencies in this environment? Comparing the decentralized equilibrium with the planner's solution shed light on this matter.

Figure 10 shows how the decentralized equilibrium relates to the planner's solution. To the right of $\theta^{*}$, agents are willing to pick action 1 , and to the left of $\theta^{*}$, action 0 . The planner would mandate that action 1 is chosen whenever the economy is to the right of $\theta^{P}$, 
instead. We can think of a vertical line crossing $\left(\theta^{*}(0.5), 0.5\right)$ as a dividing line between the regions where network 0 or network 1 are "intrinsically better". When $n=0.5$, $\theta^{*}=-\gamma / 2$, so along that vertical line $\theta^{1}=\theta^{0}$.

Figure 10: Planner's solution with linear utilities

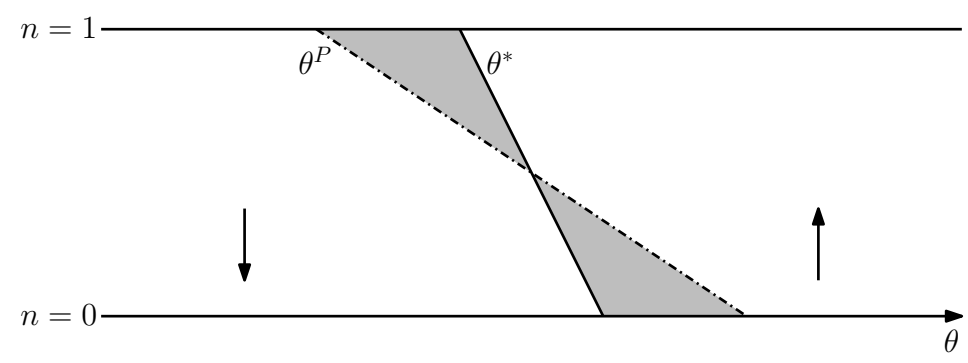

The curve $\theta^{P}$ is half as steep as $\theta^{*}$. The reason is that, in a decentralized economy, an agent at a revision opportunity considers the intrinsic quality of the two standards and the size of each network only to the extent that network effects affect her own payoff. The social planner dictating an agent's choice takes into account all factors that the agent considers plus the effect of that choice on other people. Hence, the planner will place higher weight on the coordination with the majority than the agent. Since the net externality from choosing action 1 is positive if (and only if) $n>0.5$, the planner's threshold rotates around the agent's threshold so that if $n<0.5$, the planner's threshold lies to the right of the agents', and if $n>0.5$, it lies to the left.

As a consequence, the social planner is more conservative than agents regarding the transition to a better but smaller network. There exist quality differences such that the transition to the best standard (Dvorak) happens in the decentralized economy, but the social planner would choose to stay with the worst one (QWERTY). The shaded area represents such states where inefficient shifts to the intrinsecally best network happen. If we observe that the transition to Dvorak has not happened in the decentralized economy, it must be that such transition would be inefficient (and thus should not be subsidized). The model predicts that whenever the prevailing standard is the worst-quality one, this is surely efficient.

Common wisdom would tell us that the economy gets inefficiently stuck at low-quality networks. The results show this intuition is misleading and highlight the importance of the transition costs. 


\subsubsection{Asymmetric network effects}

Now, consider the case with $\nu^{1} \neq \nu^{0}$. The planner's threshold is given by (18). When the network effect is asymmetric, the planner not only rotates the threshold around $n=0.5$, but it also shifts the threshold in order to enlarge the region in which agents choose the action that generates more externalities.

The next figure depicts the planner's solution when externalities are larger in network 0 than in network 1 . When $\nu^{0} / \nu^{1}>(\rho+\delta) / \delta$, the case of Figure 11a, the externality in network 0 is so large in comparison to the externality in 1 that the planner's threshold lies completely to the right of the agents' threshold. ${ }^{10}$ The smaller the value of $n$, the larger the quality gap needed for the switch to network 1 to be efficient. This is because the planner takes into account that, when $n$ is small, a lot of agents are stuck in action 0 (due to timing frictions) and they all would benefit from the network effects generated by an additional decrease in $n$.

Figure 11 b depicts the case with $(\rho+\delta) / \delta \geq \nu^{0} / \nu^{1}>1$. Again, the region where the planner chooses network 0 is enlarged in comparison to the case with symmetric network effects, but in this case the planner's threshold crosses the agents' at some $n>0.5$.

Figure 11: Asymmetric network effects

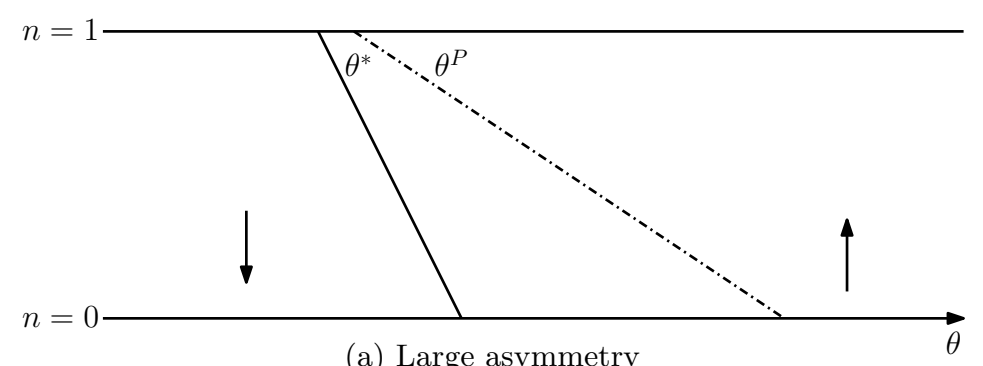

(a) Large asymmetry

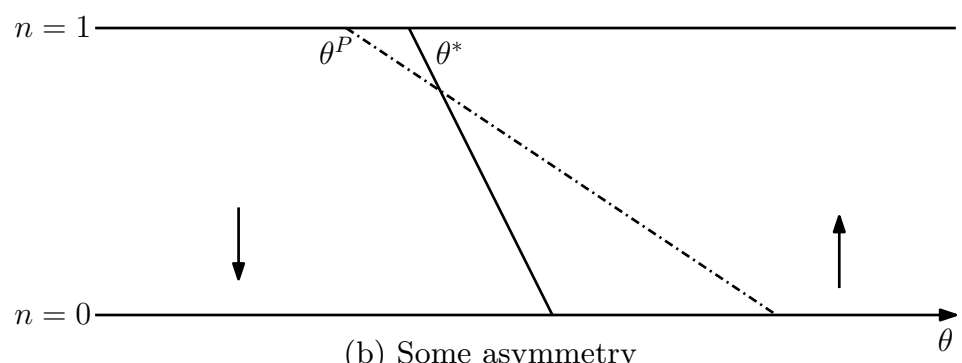

(b) Some asymmetry

${ }^{10}$ This is the case when $\theta^{P}(1)>\theta^{*}(1)$. 


\subsubsection{Alternative interpretation: negative externalities}

This result is also applicable to a case where a certain action creates negative externalities, but those externalities end up generating strategic complementarities. Consider the following variation of the model: flow-utilities from states 0 and 1 are given by

$$
u_{t}^{0}\left(\theta_{t}^{0}, n_{t}\right)=\theta_{t}^{0}-\nu^{0} n_{t} \text { and } u_{t}^{1}\left(\theta_{t}^{1}, n_{t}\right)=\theta_{t}^{1}-\nu^{1} n_{t}
$$

with $\nu^{0}>\nu^{1} \geq 0$. The relative payoff function is given by

$$
\Delta u\left(\theta_{t}, n_{t}\right)=\theta_{t}+\gamma n_{t},
$$

with $\theta_{t} \equiv \theta_{t}{ }^{1}-\theta_{t}{ }^{0}$ and $\gamma \equiv \nu^{0}-\nu^{1}>0$. These payoffs capture a situation in which action 1 harms everyone, but it is even more prejudicial to agents currently choosing action 0 . As an example, imagine that agents can adopt an aggressive behavior (action 1) or an accommodating behavior (action 0). Action 1 is particularly harmful for those adopting an accommodating behavior. ${ }^{11}$

Solving the planner's problem in this case yields the same threshold as in (18), with the feature that $\theta^{P}(1)>\theta^{*}(1)$ as in Figure 11a. Agents do not internalize the harm imposed on others when they opt for an aggressive behavior. A larger number of agents choosing an accommodating behavior (a smaller $n$ ) implies a larger potential damage of an additional agent choosing to behave aggressively, and thus the larger the gap in fundamentals required for action 1 to be the right choice from a social perspective.

\subsection{Statistical discrimination}

This section shows a simplified version of the model of statistical discrimination in Levin (2009). Individuals enter the economy at rate $\delta$ and decide whether or not to make an investment and become skilled. The cost of investment $C=-\theta$ is known at the time of the investment, where $\theta$ follows a Brownian motion.

Then, when a Poisson event occurs (again, rate $\delta$ ), an individual is matched to an employer and receives her payoff. Employers observe (i) a noisy signal about the individual's skill; and (ii) the fraction $n_{t}$ of skilled individuals in the population. The signal about the individual's skill might be 'good' or 'bad'. The signal is 'good' with probability $\phi_{H}$ if the individual is skilled and $\phi_{L}$ if she is not, with $\phi_{H}>\phi_{L}$. We will assume that skilled individuals always receive a good signal, i.e., $\phi_{H}=1$. An individual's payoff is equal to

\footnotetext{
${ }^{11}$ As an illustration, imagine that $n$ is the proportion of drivers driving SUVs, and $1-n$ is the proportion driving sedans and smaller cars. More SUVs means more severe car crashes, especially for sedan owners.
} 
the probability attached to her being skilled. When the Poisson event hits, the individual receives her payoff and leaves the model.

The investment cost $-\theta$ can be negative. One interpretation is that $\theta$ is the expected benefit from investment received after the employment opportunity considered in the model net of the investment cost.

\subsubsection{Equilibrium}

The probability an employer assigns to an individual being skilled is

$$
\gamma_{G t}=\frac{\phi_{H} n_{t}}{\phi_{H} n_{t}+\phi_{L}\left(1-n_{t}\right)} \quad \text { and } \quad \gamma_{B t}=\frac{\left(1-\phi_{H}\right) n_{t}}{\left(1-\phi_{H}\right) n_{t}+\left(1-\phi_{L}\right)\left(1-n_{t}\right)}
$$

if the signal is 'good' and if it is 'bad', respectively.

The gain from investing is the increase in the probability of getting a good signal $\left(\phi_{H}-\phi_{L}\right)$ times the present value of the gain from having a good signal $\left(\gamma_{G t}-\gamma_{B t}\right)$. Hence an individual will decide to make the investment if

$$
\left(\phi_{H}-\phi_{L}\right) E \int_{\tau}^{\infty} e^{-(\rho+\delta)(t-\tau)} \delta\left(\gamma_{G t}-\gamma_{B t}\right) d t \geq-\theta
$$

Note that both $\gamma_{G t}$ and $\gamma_{B t}$ are increasing in $n_{t}$. Intuitively, if $n_{t}$ is small, it is more likely that a good signal is due to luck and it is more likely that a bad signal reflects the individuals' true level of skill. Thus the difference $\gamma_{G t}-\gamma_{B t}$ is not necessarily increasing in $n_{t} \cdot{ }^{12}$ The assumption that $\phi_{H}=1$ ensures that the payoff from investing is increasing in $n_{t}$ because it implies $\gamma_{B t}=0$, meaning that individuals with a bad signal are surely unskilled. Individuals with a good signal might be either skilled or lucky (unskilled with a good signal) and the probability of the former is increasing in $n_{t}$. Hence agents' decisions are strategic complements. An individual will invest if:

$$
E \int_{\tau}^{\infty} e^{-(\rho+\delta)(t-\tau)} \delta \frac{n_{t}\left(1-\phi_{L}\right)}{\phi_{L}+n_{t}\left(1-\phi_{L}\right)} d t \geq-\theta
$$

Although the structure of this model is different from the framework presented in Section 2, mathematically this model can be seen as a particular case of that one. There is always a measure-one continuum of agents in the model, as agents enter and leave the economy at rate $\delta$. Agents' decisions are given by (20), which is a particular case of (4) and the flow payoff is increasing in $n_{t}$. It is easy to throw $\theta$ inside the integral to make the flow payoff increasing in $\theta$ as well and, finally, $\theta$ follows a Brownian motion.

\footnotetext{
${ }^{12}$ For example, suppose that $\phi_{H}>\phi_{L}=0$. Then $\gamma_{G t}=1$ and $\gamma_{B t}$ is increasing in $n_{t}$. Intuitively, an individual with a good signal must be skilled, but an individual with a bad signal might be either unskilled or unlucky, and the probability of the latter increases with $n_{t}$. In this case, $\gamma_{G t}-\gamma_{B t}$ is decreasing in $n_{t}$.
} 
Agents' decisions are thus determined by a unique downward sloping threshold $\theta^{*}$. Levin (2009) highlights that for some values of $\theta$, individuals would be investing if $n_{t}$ were large, but may get stuck in a situation with low $n_{t}$, reflecting the persistence of statistical discrimination: employers are likely to believe that a good signal is likely to reflect luck, not skill, and individuals refrain from investing.

The model captures a situation where the rewards from investment depend on whether others believe the investment was indeed undertaken and this belief is affected by the choices of others in the population (or, in a particular group). Beyond labor markets, this model could be applied to other problems where statistical discrimination is an issue.

\subsubsection{Efficiency}

Is the persistence of statistical discrimination inefficient? Should we be particularly concerned with economies with very low $n_{t}$ but relatively high $\theta_{t}$ ?

Using the results derived in Section 2.3, we can ask how the decentralized equilibrium compares to the planner's solution. Consider a social planner whose flow benefit from investment is given by the sum of payoffs of agents that leave the game at a given point in time. This flow benefit is then given by:

$$
W\left(n_{t}\right)=\delta\left(n_{t} \gamma_{G t}+\left(1-n_{t}\right) \phi_{L} \gamma_{G t}\right)=\delta n_{t}
$$

Intuitively, at a given moment of time, the average payoff of agents will be $n_{t}$. If signals are perfectly informative $\left(\phi_{L}=0\right)$, skilled agents (that amount to a proportion $n_{t}$ of individuals) will receive 1 and unskilled agents will receive 0 . Less informative signals imply a lower payoff for skilled agents and a larger payoff for unskilled ones, but the average payoff is still the fraction of skilled agents, $n_{t}$. Hence, the planner faces no coordination problem. An extra individual investing implies a gain equal to 1 , that is distributed among the population.

Following the steps from Section 2.3, we get that the planner chooses to invest if

$$
E \int_{\tau}^{\infty} e^{-(\rho+\delta)(t-\tau)} \delta d t \geq-\theta
$$

Note that this is exactly what we would obtain from (20) in case $\phi_{L}=0$. If $\phi_{L}=0$ and $\phi_{H}=1$, the signal about an individual is perfectly informative, so perceptions about an agent are not affected by the characteristics of the population (or group). Hence, an individual's action entails no externality.

The planner's threshold $\theta^{P}$ is given by

$$
\theta^{P}=-\frac{\delta}{\delta+\rho}
$$


Investment is efficient as long as the expected present value of its benefit is larger than the cost $-\theta$. In the $(\theta, n)$-space, the planner chooses according to a vertical threshold $\left(\theta^{P}\right.$ is independent of $n_{t}$ ).

\subsubsection{Example}

Using (13), we get the equilibrium threshold $\theta^{*}$ in the limit of vanishing shocks $(\theta \rightarrow 0)$ :

$$
\theta^{*}\left(n_{0}\right)=-\int_{0}^{n_{0}}\left(\frac{v}{n_{0}}\right)^{\frac{\rho}{\delta}} \frac{\left(1-\phi_{L}\right) v}{\phi_{L}+v\left(1-\phi_{L}\right)} d v-\int_{n_{0}}^{1}\left(\frac{1-v}{1-n_{0}}\right)^{\frac{\rho}{\delta}} \frac{\left(1-\phi_{L}\right) v}{\phi_{L}+v\left(1-\phi_{L}\right)} d v
$$

Figure 12 shows an example with $\rho=0.1, \delta=0.2$. The solid vertical line at the left is the planner's threshold, from (21). The other curves are the equilibrium thresholds for $\phi_{L}=0.1, \phi_{L}=0.5$ and $\phi_{L}=0.9$, starting from the left.

Figure 12: Equilibrium in case of vanishing shocks

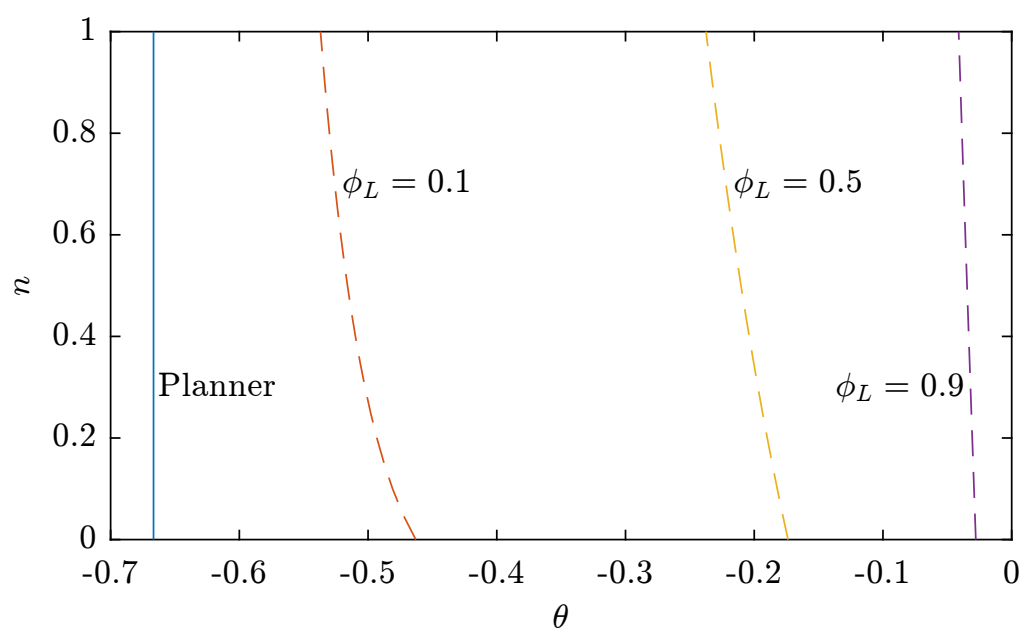

When $\phi_{L}=0.9$, the private benefit from investing is small, as unskilled agents are likely to get a good signal. Hence, in equilibrium, investment pays off only if its cost is close to zero. Interestingly, even when the signal is very informative, there are important inefficiencies. The leftmost dashed curve corresponds to the case $\phi_{L}=0.1$ (and, as in all other cases, $\phi_{H}=1$ ). Still, the equilibrium threshold is quite far from the efficient benchmark, especially for low $n$. When $n=0$, agents are willing to pay no more than 0.46 for investing, but it would be efficient to pay up to 0.67 . When $n=1$, agents are willing to pay up to 0.57 .

In sum, the persistence of statistical discrimination is inefficient and we should indeed be particularly concerned with economies with very low $n_{t}$ but relatively high $\theta_{t}$. 


\subsection{A macroeconomic model}

Kiyotaki (1988) shows that in a model with monopolistic competition and some locally increasing returns to scale, there are multiple equilibria. Guimaraes and Machado (2017) embed a simple model that captures those insights in a dynamic framework with timing frictions.

The demand side is standard. A continuum of risk-neutral agents produce each a differentiated good that are aggregated by a competitive final good producer according to:

$$
Y_{t}=\left(\int_{0}^{1} y_{i, t}^{\frac{\varepsilon-1}{\varepsilon}} d i\right)^{\frac{\varepsilon}{\varepsilon-1}}
$$

where $Y_{t}$ is the amount produced of the final good, $y_{i, t}$ is the amount purchased of the variety produced by agent $i$, and $\varepsilon>1$ is the elasticity of substitution. The zero profit condition for final good producers is

$$
\int_{0}^{1} p_{i, t} y_{i, t} d i=P Y,
$$

where $p_{i, t}$ is the price of variety $i$.

The intermediate good producer can operate in two regimes: 1 (High) and 0 (Low). Chances to switch regimes arrive according to a Poisson process with arrival rate $\delta$. Agents in the High regime can produce up to $e^{\theta_{t}} x_{H}$ and agents in the low regime can produce up to $e^{\theta_{t}} x_{L}$, with $x_{H}>x_{L}$. The marginal cost is zero up to firms' capacity, but firms must pay a fixed cost $\psi>0$ to choose the High regime when they get the chance. One should think of moving to regime High as buying some machine that decreases the marginal cost of production. When a producer in regime High gets the Poisson realization, the machine fully depreciates and she gets a chance to get another one. When a producer in regime Low gets the Poisson realization, it simply means that an investment opportunity has arrived.

Alternatively, one could say that producers rent a machine but must pay a competitive user cost $\tilde{\psi}=(\rho+\delta) \psi$ that equals the expected depreciation $(\delta \psi)$ plus interest $(\rho \psi)$. Since agents are risk-neutral, as long as they can borrow in frictionless credit markets, they are indifferent between buying or renting and both interpretations are equivalent.

\subsubsection{Equilibrium}

Let $P_{t}$ be the price of the final good. Say producers of the final good will spend $C$ on intermediate goods. At every instant, they will choose inputs $y_{i}$ in order to maximize (22) 
subject to

$$
\int_{0}^{1} p_{i, t} y_{i, t} d i=C
$$

taking prices of intermediate goods as given. This is a static constrained-maximization problem. Combining the first-order conditions for intermediate goods $i$ and $j$ and rearranging yield

$$
\frac{p_{i}}{p_{j}}=\left(\frac{y_{i}}{y_{j}}\right)^{-1 / \varepsilon} .
$$

Plugging this expression into the zero-profit condition in (23) yields the usual demand for each variety:

$$
p_{i, t}=\left(\frac{y_{i, t}}{Y_{t}}\right)^{\frac{1}{\varepsilon}} P_{t} .
$$

Using (24), and the fact that it will always be optimal to produce at maximum capacity, we can write the profit of producers in regime High and Low as

$$
u_{1}=p_{i, t} y_{i, t}-\tilde{\psi}=\left(e_{t}^{\theta} x_{H}\right)^{\frac{\varepsilon-1}{\varepsilon}} Y_{t}^{\frac{1}{\varepsilon}} P_{t}-\tilde{\psi}
$$

and

$$
u_{0}=p_{i, t} y_{i, t}=\left(e_{t}^{\theta} x_{L}\right)^{\frac{\varepsilon-1}{\varepsilon}} Y_{t}^{\frac{1}{\varepsilon}} P_{t}
$$

By market clearing we have

$$
Y_{t}=\left(n_{t}\left(e^{\theta_{t}} x_{H}\right)^{\frac{\varepsilon-1}{\varepsilon}}+\left(1-n_{t}\right)\left(e^{\theta_{t}} x_{L}\right)^{\frac{\varepsilon-1}{\varepsilon}}\right)^{\frac{\varepsilon}{\varepsilon-1}} .
$$

Plugging (27) into (25) and (26), and normalizing $P_{t}$ to 1 , we get:

$$
u_{1}\left(\theta_{t}, n_{t}\right)=e^{\theta_{t}} x_{H}^{\frac{\varepsilon-1}{\varepsilon}}\left(n_{t} x_{H}^{\frac{\varepsilon-1}{\varepsilon}}+\left(1-n_{t}\right) x_{L}^{\frac{\varepsilon-1}{\varepsilon}}\right)^{\frac{1}{\varepsilon-1}}-\widetilde{\psi}
$$

and

$$
u_{0}\left(\theta_{t}, n_{t}\right)=e^{\theta_{t}} x_{L}^{\frac{\varepsilon-1}{\varepsilon}}\left(n_{t} x_{H}^{\frac{\varepsilon-1}{\varepsilon}}+\left(1-n_{t}\right) x_{L}^{\frac{\varepsilon-1}{\varepsilon}}\right)^{\frac{1}{\varepsilon-1}},
$$

which implies that

$$
\Delta u\left(\theta_{t}, n_{t}\right)=e^{\theta_{t}}\left(n_{t} x_{H}^{\frac{\varepsilon-1}{\varepsilon}}+\left(1-n_{t}\right) x_{L}^{\frac{\varepsilon-1}{\varepsilon}}\right)^{\frac{1}{\varepsilon-1}}\left(x_{H}^{\frac{\varepsilon-1}{\varepsilon}}-x_{L}^{\frac{\varepsilon-1}{\varepsilon}}\right)-\widetilde{\psi} .
$$

Notice that the expression above is increasing in $\theta_{t}$ and $n_{t}$. To guarantee the existence of dominance regions (assumption 1), we need to impose that $\sigma^{2}<2(\rho+\delta) .{ }^{13}$ Then, this model can be seen as a particular case of the framework presented in Section 2.

\footnotetext{
${ }^{13}$ This is because the integral $\int_{0}^{\infty} e^{-(\rho+\delta) t} \mathbb{E}\left[e_{t}^{\theta}\right] d t=\int_{0}^{\infty} e^{-\left(\rho+\delta-0.5 \sigma^{2}\right) t} e^{\theta_{0}} d t$ diverge to $\infty$ if $\rho+\delta-0.5 \sigma^{2}<0$, making it always optimal to choose regime High.
} 
Firms have higher incentives to invest if they expect others to do the same in the future. This is so because of the demand externality: when others increase their production, the demand for one's variety increases, as shown in (24). Hence firms face a dynamic coordination problem in their investment decisions.

In what follows, it is useful to define $\widetilde{\Delta u}(\theta, n) \equiv \Delta u(\theta, n)+\widetilde{\psi}$. Notice that $\widetilde{\Delta u}(\theta, n)$ is simply the instantaneous gain in revenue of operating in regime High instead of Low. The instantaneous losses from higher costs is $\widetilde{\psi}$. Using (4), agents choose to invest as long as:

$$
\int_{\tau}^{\infty} e^{-(\rho+\delta)(t-\tau)} \mathbb{E}_{\tau}\left[\widetilde{\Delta u}\left(\theta_{t}, n_{t}\right)\right] d t \geq \psi
$$

The equilibrium in given by the unique threshold $\theta^{*}$ that satisfies (28).

The dynamic coordination problem faced by firms may lead to dynamic inefficiencies that persist over time: firms may not invest today because they are not confident others will invest tomorrow. But that contributes to lower investment tomorrow, since economic activity will be low. In other words, the economy may get in a dynamic coordination trap.

\subsubsection{Optimal stimulus policies}

Some natural questions that emerge are the following: what kind of investment subsidies can implement the first-best? Does the dynamic coordination problem imply that a social planner should provide counter-cyclical subsidies, i.e., higher subsidies in times of low economic activity (low $n_{t}$ )? We can use this model to answer these questions.

Using (6), (25), (26) and (27), the social planner instantaneous payoff is given by

$$
W(\theta, n)=Y_{t}-\widetilde{\psi} n_{t}
$$

which yields

$$
\frac{d W(\theta, n)}{d n}=\left(\frac{\varepsilon}{\varepsilon-1}\right) \widetilde{\Delta u}\left(\theta_{t}, n_{t}\right)-\widetilde{\psi}
$$

As shown in Section 2.3, the social planner's decision threshold must satisfy (5). The planner thus chooses to invest as long as:

$$
\int_{\tau}^{\infty} e^{-(\rho+\delta)(t-\tau)} \mathbb{E}_{\tau}\left[\left(\frac{\varepsilon}{\varepsilon-1}\right) \widetilde{\Delta u}\left(\theta_{t}, n_{t}\right)-\widetilde{\psi}\right] d t \geq 0 .
$$

Rearranging and using $\widetilde{\psi}=(\rho+\delta) \psi$ yield

$$
\int_{\tau}^{\infty} e^{-(\rho+\delta)(t-\tau)} \mathbb{E}_{\tau}\left[\widetilde{\Delta u}\left(\theta_{t}, n_{t}\right)\right] d t \geq \psi-\frac{1}{\varepsilon} \psi .
$$


This is very similar to (28). It implies that the agents' problem is identical to the planner's problem if they get an investment subsidy of $\frac{1}{\varepsilon} \psi$. The planner can therefore implement the first-best by providing a constant investment subsidy. Alternatively, it could pay $\frac{1}{\varepsilon-1}$ dollars for each unit of revenue firms get (equation 30). The important thing here is that the planner is not more willing to incentivize investment in times of low economic activity.

Now notice that we can write agents' payoffs as $\Delta u\left(\theta_{t}, n_{t}\right)=e^{\theta_{t}} g\left(n_{t}\right)-\widetilde{\psi}$. Therefore, the planner's flow payoff can be written as:

$$
\frac{d W(\theta, n)}{d n}=e^{\theta_{t}+\log \left(\frac{\varepsilon}{\varepsilon-1}\right)} g\left(n_{t}\right)-\widetilde{\psi}
$$

Let $b_{t}=\theta_{t}+\log \left(\frac{\varepsilon}{\varepsilon-1}\right)$. Notice that $b_{t}$ follows the same law of motion as $\theta_{t}$. Thus the planner's problem is identical to the agent's problem if the fundamental is $b_{t}$ instead of $\theta_{t}$. Hence, the planner's threshold plotted in the $\left(b_{t}, n_{t}\right)$-space is identical to the agent's threshold plotted in the $\left(\theta_{t}, n_{t}\right)$-space, which implies that the planner's threshold is a left translation of the agent's threshold, and the distance between the two is $\log \left(\frac{\varepsilon}{\varepsilon-1}\right)$. This result is shown in Figure 13.

Figure 13: Planner's translation

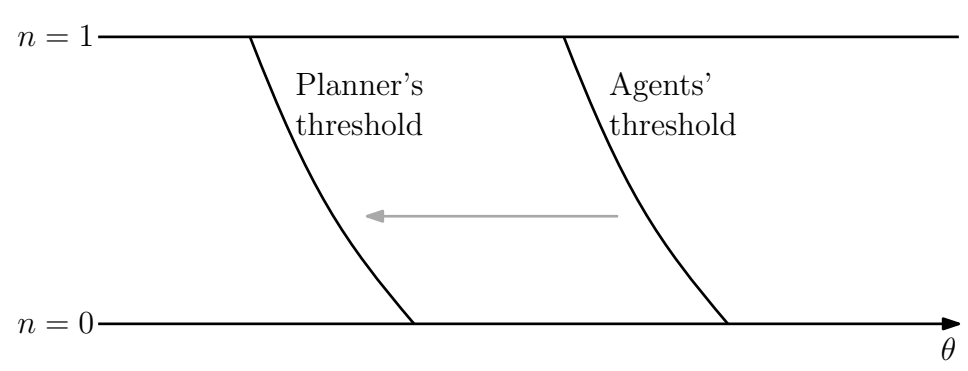

A similar reasoning implies that the distance between the planner's and the agents' threshold (for a given $n$ ) is proportional to the maximum subsidy the planner is willing to pay (which is the subsidy required to make agents invest on the planner's threshold). A subsidy of $x$ units is equivalent to increasing $\theta$ in $\log (1-x / \psi)$.

Agents always benefit if others invest (action 1), regardless of their current action. Using (9), (29) and $\Delta u(\theta, n)=\widetilde{\Delta u}(\theta, n)-\widetilde{\psi}$, we get that the externality term is always positive (and proportional to $\widetilde{\Delta u}(\theta, n)$ ). The planner always has higher incentives to choose action 1 and thus chooses a threshold that lies entirely to the left of the agent's threshold.

But why does not the dynamic coordination problem require higher subsidies in times of low economic activity? To better understand the results it is useful to look at the planner's problem in a world without shocks and with multiple equilibria. 


\subsubsection{Optimal stimulus with no shocks}

The solution to the planner's problem when $\sigma \rightarrow 0$ is the same as the solution with $\sigma=0$. But we know that the agent's problem is very different. Figure 14 combines the results depicted in Figure 8 and Figure 13, showing the agents' threshold with $\sigma \rightarrow 0\left(\theta^{*}\right)$, the two extreme equilibria of the game without shocks $\left(\theta^{\text {opt }}\right.$ and $\left.\theta^{\text {pes }}\right)$ and the planner's threshold.

Figure 14: Planner's problem when $\sigma=0$

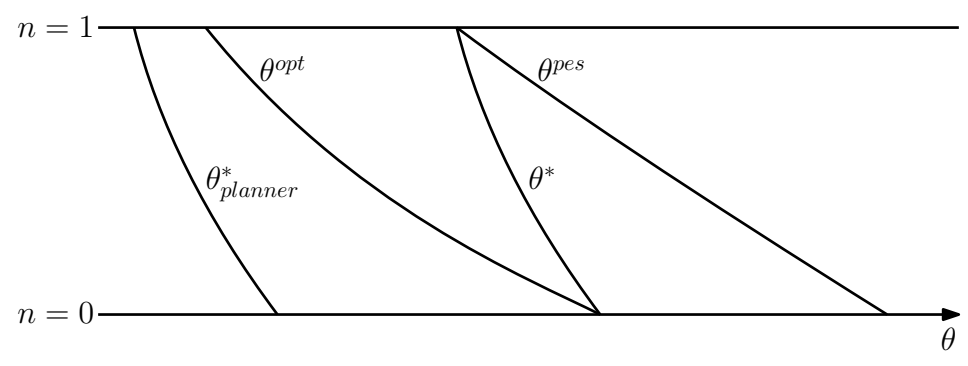

When agents play according to either $\theta^{\text {opt }}$ or $\theta^{\text {pes }}$, the distance between the planner's and the agents' threshold is larger when $n=0$ than when $n=1$. Therefore, regardless of whether we choose the 'good' or 'bad' equilibrium, the model with multiple equilibria predicts that the planner is more willing to pay subsidies in times of low economic activity, while the model with a unique equilibrium prescribes a constant subsidy.

Figure 15 illustrates the intuition for this result. First, imagine we are in a world where agents play according to the good equilibrium. In that case, it is harder to coordinate when $n=0$ for an intuitive reason. In times of low economic activity but reasonably good fundamentals everyone would be happy to sign a contract that forces everyone to invest, even though the instantaneous gains of doing so today are negative. This is because higher investment will force a regime switch that will benefit everyone in the future. The problem is that no one wants to be the first to invest, leading to a dynamic coordination trap where one firm keeps waiting for the other to move. This is reflected by the negative association between $n$ and the distance between the planner's threshold and the good equilibrium in Figure 15. We dub it the dynamic problem.

Now suppose the economy is at some point between $\theta^{\text {opt }}$ and $\theta^{\text {pes }}$ and for some reason agents are not investing. We know that in that region agents would invest if they were as optimistic as possible. Thus, whenever agents are not investing in the region between $\theta^{\text {opt }}$ and $\theta^{\text {pes }}$ we say that there is a beliefs problem. For instance, if agents play according to the unique equilibrium with vanishing shocks the size of the beliefs problem for a given $n$ is the gray area in Figure 15. Notice that $\theta^{*}$ touches the good equilibrium when $n=0$ 
and the bad equilibrium when $n=1$.

When agents play according to the unique equilibrium with vanishing shocks, the dynamic problem is higher when $n=0$, but the beliefs problem is non-existent. Conversely, when $h=1$ there is no dynamic problem, but the beliefs problem is very severe. It turns out that the different size of the beliefs problem across different values $n$ exactly cancels the dynamic problem. In the equilibrium with small shocks, agents are very optimistic at the threshold when $n=0$ and very pessimistic when $n=1$. Why?

Consider an agent deciding on the threshold $\theta^{*}$ when $n=0$. She knows that if negative shocks hit, that will not change the level of economic activity. But if positive shocks hit, the economy will bifurcate up and leave the investment slump. Good shocks create a boom, while bad shocks do not worsen economic activity. Conversely, when $n=1$ and the economy is at the threshold $\theta^{*}$, good shocks do not increase economic activity, while bad shocks trigger an investment slump. The beliefs that arise in equilibrium offset the dynamic coordination trap, eliminating the need for higher subsidies at times of low economic activity.

Figure 15: Dynamic problem and beliefs problem $\sigma=0$

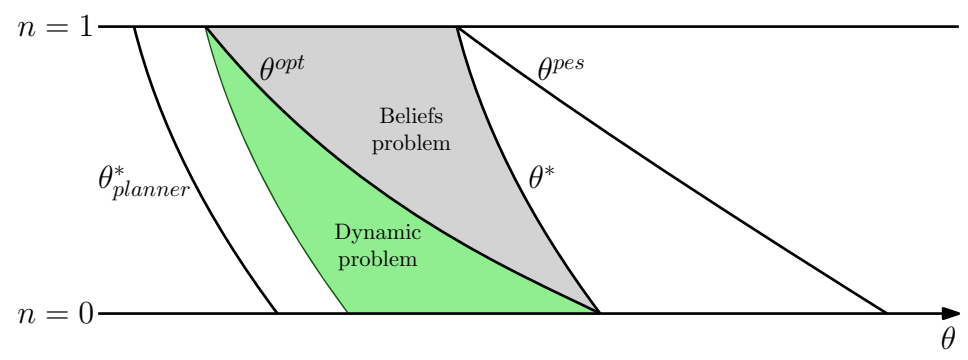

\subsubsection{Numerical solution}

In this section we discuss some of the positive implications of the model of Guimaraes and Machado (2017) through some numerical examples. The goal here is not to provide an extensive quantitative analysis, but only to illustrate how this class of models can be used by macroeconomists interested in business cycles. First, it will be shown how to solve the model numerically. Then, through some simple simulations, we discuss some properties of the model.

Before solving the model we make a departure from our baseline framework. Instead of assuming the $d Z_{t}$ follows a Brownian motion, we assume that it follows a mean reverting Ornstein-Uhlenbeck process:

$$
d \theta_{t}=\eta\left(\mu-\theta_{t}\right) d t+d Z_{t}
$$


with $\eta$ and $\sigma$ larger than zero. When $\eta=0$ we are back to the case studied throughout this survey. The parameter $\eta$ represents the strength of mean reversion on the process for $\theta_{t}$. The parameter $\mu$ represents the mean it reverts to. Mean reversion is a desirable property when taking this kind of model to the data, so that cycles become frequent. Notice that our proof for uniqueness does not work in that case. Fortunately, Frankel and Burdzy (2005) show that under some additional technical assumptions, the equilibrium is still unique with mean reversion. Moreover, even without additional assumptions, numerically one can verify that if there is multiplicity, it does not seem to matter quantitatively.

Discrete approximation. In order to solve the model numerically, we work with an approximation of the model. Now time is discrete and each period has length $\Delta$, where $\Delta$ is a small number. Hence $t \in\{0, \Delta, 2 \Delta, 3 \Delta, \ldots\}$. The stochastic process of $\theta_{t}$ is given by

$$
\theta_{t}=\theta_{t-1}+\left(\mu-\theta_{t-1}\right) \Delta+\sigma \sqrt{\Delta} \epsilon_{t}
$$

where $\epsilon_{t}$ are iid normal shocks with zero mean and unit variance. At the beginning of each period, after $\theta_{t}$ is observed, $\left(1-e^{-\delta \Delta}\right)$ individuals are randomly selected and get a chance to switch regime. Agents discount their utility between two periods by the factor $e^{-\rho \Delta t}$. When $\Delta \rightarrow 0$ this model converges to the continuous time model.

Threshold computation. Our goal is to find a threshold where agents are indifferent between actions 1 and 0 if they believe others will play according to that threshold. The steps are basically the following:

1. Pick an arbitrary threshold $\theta_{0}^{*}$ and choose a finite grid for $n$ in the interval $[0,1]$.

2. For every point $n$ in the grid, simulate $N$ paths of $\theta_{t}$ and $n_{t}$ departing from $\left(\theta_{0}^{*}(n), n\right)$, assuming every agent will play according to $\theta_{0}^{*}$. Use those paths to estimate the gain in utility from choosing action 1 of an agent choosing at $\left(\theta_{0}^{*}(n), n\right)$.

3. If the gain in utility is close to zero in every point of the grid, stop. Otherwise, update $\theta_{0}^{*}$ and go back to step 2 .

Alternatively, we can assume every agent is choosing action 0 and find the threshold that determines the region where choosing 1 is a dominant strategy (call it $\theta_{0}^{H}$ ). Then assume all agents play according to $\theta_{0}^{H}$, find again the best response and keep iterating until it converges. Of course, we could start the iterations assuming everyone chooses action 1 in the first iteration. 
Numerical results. We illustrate the workings of the model here through a numerical example. ${ }^{14}$ Figure 16 shows the computed threshold. The dotted line represents a path for $\left(\theta_{t}, n_{t}\right)$.

Figure 16: Estimated threshold

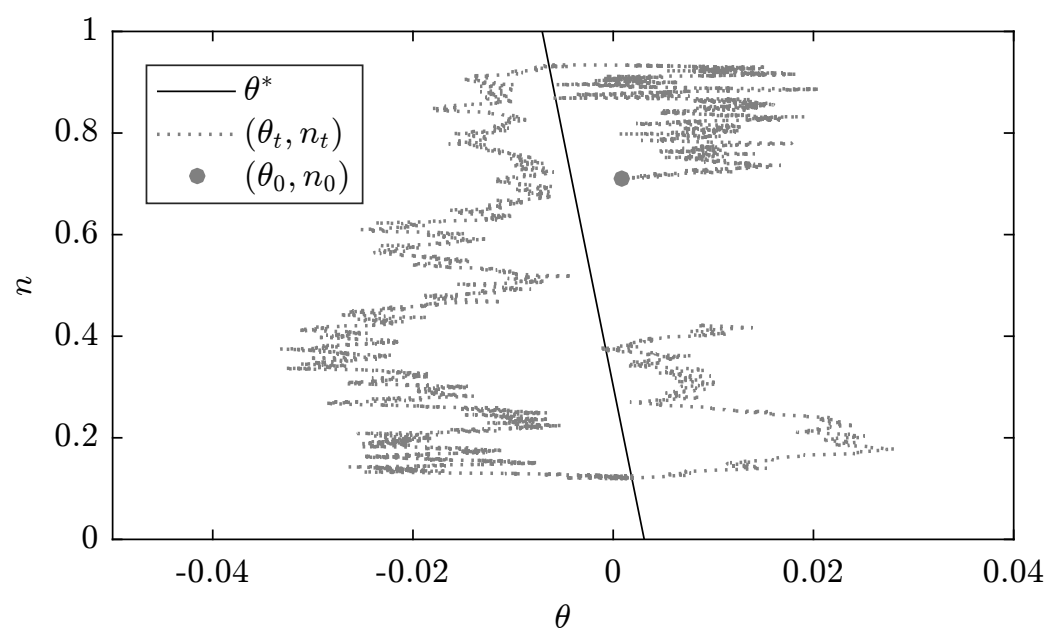

Note that the threshold is to the right of $\theta=0$ when $n=0$ and to to left when $n=1$. It means that at the long-run average of $\theta_{t}$, which is zero in this example, the economy can be either to the right or to the left of the threshold, depending on agents' past decisions (which are summarized by $\left.n_{t}\right)$. Thus, at the point $(\theta, n)=(0,0)$, the economy will not leave the investment slump unless sufficiently good shocks hit. Similarly, at the point $(\theta, n)=(0,1)$, sufficiently negative shocks are needed to trigger a regime change.

Figure 17 shows the output dynamics in this economy. The economy is simulated using a sequence of random shocks $d Z_{t}$, starting at a point in which $n_{0}=1$ and $\theta_{0}=\mu=0$ (the 'steady-state' of $\theta_{t}$ ). The solid line represents the log deviations of output relative to its initial value. The dotted line represents the deviations of productivity $\theta_{t}$, which would be equivalent to the log deviations of output if firms always operated in regime High (note that in regime High output is $\left.e^{\theta_{t}} x_{H}\right)$.

Two important messages come from this figure. First, when shocks are large, the coordination mechanism amplifies the shocks to productivity in this economy, as reflected by the higher fall in output after sufficiently bad shocks. Second, the model generates slow recoveries. Productivity returns to its initial level much earlier than output. This illustrates the potential of this kind of framework to generate deep recessions and slow recoveries.

\footnotetext{
${ }^{14}$ The parameters used are $\varepsilon=4, x_{H}=1.05, x_{L}=1, \delta=1, \psi=0.0364, \sigma=0.03, \rho=0.03, \eta=0.7, \mu=0$.
} 
Figure 17: Amplification and slow recovery

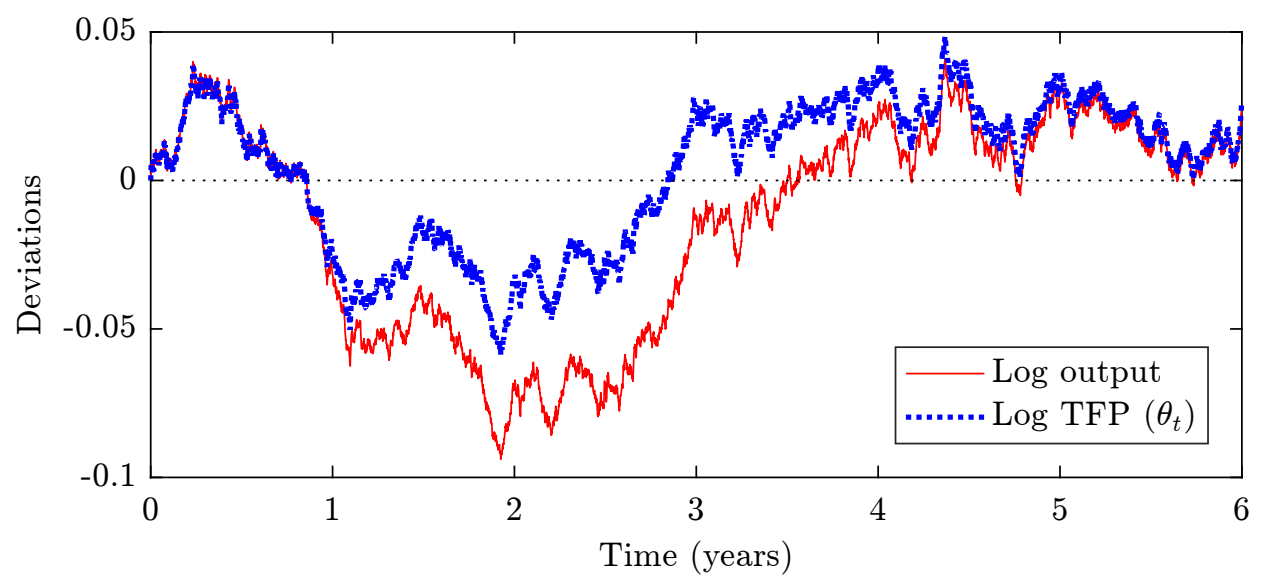

\subsection{Housing and coordination}

The basic framework considers a continuum of agents with measure one. However, it also fits cases where there is a fixed supply of positions and two large groups of agents that bid for those positions.

In the model of Frankel and Pauzner (2002), there is a measure-one continuum of houses in a neighborhood and a larger measure of agents of two groups (they call them 'blacks' and 'whites', we will call them 0 and 1). An agent from group $i \in\{0,1\}$ living in the neighborhood gets a flow utility $u_{i}\left(\theta_{t}, n_{t}\right)$ where $\theta_{t}$ indicates the relative attractiveness of the neighborhood for people in group 1 (the attractiveness of other neighborhoods is normalized to zero) and $n_{t}$ is the measure of houses owned by people from group 1 . It is assumed that $u_{0}$ is increasing in $\left(1-n_{t}\right)$ and $u_{1}$ is increasing in $n_{t}$.

People living in the neighborhood get moving opportunities at rate $\delta$ (say they have to go somewhere else). When this happens, they sell their houses to whoever offers a larger price (there is a large number of people from both groups willing to buy the house).

For an individual from group $i$ at time $\tau$, a house in the neighborhood is worth

$$
P_{\tau}^{i}=\int_{\tau}^{\infty} e^{-(\rho+\delta)(t-\tau)} \mathbb{E}\left[u_{i}\left(\theta_{t}, n_{t}\right)\right] d t+\int_{\tau}^{\infty} \delta e^{-(\rho+\delta)(t-\tau)} \mathbb{E}\left[P_{t}^{S}\left(\theta_{t}, n_{t}\right)\right] d t,
$$

The first term is the utility the agent will get while living in the house until she has to move. The second term is the expected price the agent gets when she sells the house, where $P_{t}^{S}\left(\theta_{t}, n_{t}\right)$ is the price she sells a house when the state is $\left(\theta_{t}, n_{t}\right)$. Note that the second term does not depend on the type of agent (the value of the house does not depend on the type of the seller, she has to sell it anyway).

An individual from group 1 will outbid individuals from group 0 if and only if $P_{\tau}^{1}>P_{\tau}^{0}$. 
That happens if:

$$
\int_{\tau}^{\infty} e^{-(\rho+\delta)(t-\tau)} \mathbb{E}\left[\Delta u\left(\theta_{t}, n_{t}\right)\right] d t \geq 0
$$

where $\Delta u\left(\theta_{t}, n_{t}\right)=u_{1}\left(\theta_{t}, n_{t}\right)-u_{0}\left(\theta_{t}, n_{t}\right)$. This is equivalent to the expression in (1), and since $\Delta u$ is increasing in $\theta_{t}$ and in $n_{t}$, all results from Section 2 apply here.

\section{Extensions}

Frankel and Pauzner (2000) assume that fundamentals follow a Brownian motion and allow for a deterministic drift. Frankel and Burdzy (2005) generalize the uniqueness result allowing for seasonal and mean-reverting shocks to $\theta$. Daniëls (2009) extends the uniqueness result to the case where $\theta$ follows a jump diffusion process and derives an expression analogous to (14) for the case of vanishing frictions.

Plantin and Shin (2011) extend the model to allow for payoffs that depend not only on $n_{t}$ but also on $\dot{n}_{t}$. Besides showing a uniqueness result, they use the model to study whether carry trades have a stabilizing or a destabilizing effect.

This section considers three other extensions: a model where agents choose the switching rate from a continuous set; the case with ex-ante heterogeneous agents; and an environment with coordination and preemption motives, suitable to the study of crises.

\subsection{Endogenous timing}

So far agents were not allowed to decide about the timing of their opportunities to switch actions. But in many applications this can be an important margin of adjustment. For instance, a firm looking for a worker may decide to increase its search intensity if it faces higher demand; a firm building a new plant may want to speed up its construction if the economy is booming; a consumer unsatisfied with his current cell phone may want to try to sell it on secondary markets. The framework of Frankel and Burdzy (2005) allows agents to choose the arrival rate of their switching opportunities.

Now agents choose the switching rate instead of actions 0 and 1 directly. Agents locked in action 0 choose an arrival rate $\delta^{0}$ of the Poisson process. Once the Poisson shock hits, the agent automatically switches to action 1 (she cannot choose if she wants to go). Similarly, agents locked in action 1 choose the switching rate $\delta^{1}$ of the Poisson process that will send them to regime 0 . At date $t$, an agent in regime 1 that chooses some arrival rate $\delta_{t}^{1}$ must pay a cost $c^{1}\left(\delta_{t}^{1}\right)$. Therefore, the flow payoff of this agent is given by $u_{1}\left(\theta_{t}, n_{t}\right)-c^{1}\left(\delta_{t}^{1}\right)$. Similarly, agents in regime 0 bear a $\operatorname{cost} c^{0}\left(\delta_{t}^{0}\right)$ and their flow 
payoff is $u_{0}\left(\theta_{t}, n_{t}\right)-c^{0}\left(\delta_{t}^{0}\right)$. We assume that $c^{j}(\cdot)$ weakly increasing and left-continuous in $\delta^{j}$. Moreover, $\delta_{t}^{j} \in K_{j}$, where $K_{j}$ is some closed interval in $\mathbb{R}_{+}$. The relative gain of being in regime high is

$$
D\left(\theta, n, \delta^{0}, \delta^{1}\right)=\Delta u(\theta, n)-\left[c^{1}\left(\delta^{1}\right)-c^{0}\left(\delta^{0}\right)\right] .
$$

It is assumed that $D(\cdot)$ is weakly increasing and Lipschitz in $\theta$ and $n .{ }^{15}$ Moreover, we make the assumption below, which is simply the counterpart of Assumption 1.

Assumption 2 (Existence of dominance regions). There exists $\tilde{\theta}$ and $\underset{\sim}{\theta}$ such that: if $\theta_{t}>\tilde{\theta}$, it is strictly dominant for players in regime 0 (1) to switch at the maximal (minimal) rate; if $\theta_{t}<\tilde{\theta}$ it is strictly dominant for players in regime 0 (1) to switch at the minimal (maximal) rate.

This framework can easily accommodate the baseline model. We can set $K_{0}=K_{1}=$ $[0, \delta]$ and $c^{0}\left(\delta^{0}\right)=c^{1}\left(\delta^{1}\right)=0$, for every $n, \delta^{0}$ and $\delta^{1}$. Then, choosing action $j$ with probability $p$ conditional on getting the chance in the baseline model, is equivalent to choosing an arrival rate $\delta_{t}^{j}=(1-p) \delta$ and $\delta_{t}^{1-j}=p \delta$ in this model.

Let $\Delta \mathcal{V}_{\tau}$ denote the lifetime utility of being in regime 1 minus the lifetime utility of being in regime 0 , at date $\tau$. This is given by

$$
\Delta \mathcal{V}_{\tau}=\mathbb{E}_{\tau}\left[\int_{\tau}^{\infty} e^{-\int_{\tau}^{t}\left(\rho+\delta_{t}^{0}+\delta_{t}^{1}\right) d t} D\left(\theta_{t}, n_{t}, \delta_{t}^{0}, \delta_{t}^{1}\right) d t\right]
$$

Note that $\delta_{t}^{0}$ and $\delta_{t}^{1}$ appear on the discount factor. This is because the difference in utility between agents in regime 1 and 0 becomes zero whenever both agents are in the same regime. It happens either when an agent in regime 1 switches to regime 0 ; or when an agent in regime 0 switches to regime 1 . The probability that none of those events have happened at some date $t>\tau$ is $\exp \left\{-\int_{\tau}^{t}\left(\delta_{t}^{0}+\delta_{t}^{1}\right) d t\right\}$.

As shown in Frankel and Burdzy (2005), there is a unique equilibrium in this game. At each date $\tau$, agents in regime 1 choose a switching rate $\delta^{1}=\delta^{1 *}\left(n_{t}, \theta_{t}\right)$ to maximize

$$
\delta^{1} \Delta\left(-\mathcal{V}_{\tau}\right)-c^{1}\left(\delta^{1}\right)
$$

Similarly, agents in regime 0 choose a switching rate $\delta^{0}=\delta^{0 *}\left(n_{t}, \theta_{t}\right)$ to maximize

$$
\delta^{0} \Delta \mathcal{V}_{\tau}-c^{0}\left(\delta^{0}\right)
$$

If the solution is interior, these expressions are simply stating that agents equalize the marginal cost of increasing the probability of switching with the expected gain. In equilibrium, agents take as given their own switching rate in the future and the switching rate

\footnotetext{
${ }^{15}$ Under some additional assumptions, the cost $c(\cdot)$ can also depend on $n_{t}$. See Frankel and Burdzy (2005).
} 
of others $(\Delta \mathcal{V}$ is taken as given) and simply maximize the instantaneous benefit of doing it.

\subsubsection{The planner's problem}

We add the assumption that $\operatorname{costs} c^{j}(\cdot)$ are convex. This implies that the planner chooses the same hazard rate for every agent. For simplicity, we assume a constant hazard rate for agents locked in action 1 , equal to $\delta^{1}$, with $c^{1}\left(\delta^{1}\right)=0$. Hence welfare in this economy is given by

$$
E_{\tau} \int_{\tau}^{\infty} e^{-\rho(t-\tau)} W\left(\theta_{t}, n_{t}, \delta_{t}^{0}\right) d t
$$

with

$$
W\left(\theta_{t}, n_{t}, \delta_{t}^{0}\right)=n_{t} u_{1}\left(\theta_{t}, n_{t}\right)+\left(1-n_{t}\right)\left[u_{0}\left(\theta_{t}, n_{t}\right)-c^{0}\left(\delta_{t}^{0}\right)\right]
$$

The strategy here follows Guimaraes and Machado (2017) and is similar to the employed in Section 2.3. Suppose the planner is following the optimal plan and consider the following deviation: change $\delta_{t}^{0}$ to $\tilde{\delta}$ at $\tau$ for an infinitesimal period $d t$ and keep future choices for every realization of the Brownian path in the future unchanged. This affects current costs and output net of switching costs for all $s>\tau$. Since there are $1-n_{t}$ agents locked in action 0 , costs change by

$$
\left[c^{0}(\tilde{\delta})-c^{0}\left(\delta_{t}^{0}\right)\right]\left(1-n_{t}\right) d t
$$

and the immediate effect on $n_{t}$ is

$$
d n_{\tau}=\left(\tilde{\delta}-\delta_{t}^{0}\right)\left(1-n_{t}\right) d t
$$

This effect dies out in time:

$$
d n_{s}=d n_{\tau}-\int_{\tau}^{s} d n_{v}\left(\delta_{v}^{0}+\delta^{1}\right) d v
$$

which implies that

$$
d n_{s}=d n_{\tau} e^{-\int_{\tau}^{s}\left(\delta_{v}^{0}+\delta^{1}\right) d v} .
$$

The effect on welfare $W$ net of switching costs for $s>\tau$ is

$$
E_{\tau} \int_{\tau}^{\infty} e^{-\rho(s-\tau)}\left(\frac{\partial W\left(\theta_{s}, n_{s}, \delta_{s}^{0}\right)}{\partial n_{s}} d n_{s}\right) d s
$$

This deviation cannot be profitable. Hence, putting together (32), (33), (34) and (35), it must be that

$$
\left(\tilde{\delta}-\delta_{t}^{0}\right) E_{\tau} \int_{\tau}^{\infty} e^{-\int_{\tau}^{s}\left(\rho+\delta_{v}^{0}+\delta^{1}\right) d v}\left(\frac{\partial W\left(\theta_{s}, n_{s}, \delta_{s}^{0}\right)}{\partial n_{s}}\right) d s-\left[c^{0}(\tilde{\delta})-c^{0}\left(\delta_{t}^{0}\right)\right] \leq 0,
$$


for any $\tilde{\delta} \in[0, \bar{\delta}]$. That is equivalent to stating that a necessary condition for the planner's solution is that $\delta_{t}^{0}$ must maximize

$$
\delta_{\tau}^{0} E_{\tau} \int_{\tau}^{\infty} e^{-\int_{\tau}^{s}\left(\rho+\delta_{v}^{0}+\delta^{1}\right) d v}\left(\frac{\partial W\left(\theta_{s}, n_{s}, \delta_{s}^{0}\right)}{\partial n_{s}}\right) d s-c^{0}\left(\delta_{\tau}^{0}\right)
$$

This expression is similar to (31). The difference is the term $\partial W\left(\theta_{s}, n_{s}, \delta_{s}^{0}\right) / \partial n_{s}$ inside the integral instead of $D\left(\theta_{s}, n_{s}, \delta_{s}^{0}\right)$. As in (9), the planner considers the externality on other agents. Mathematically, finding the solution to the planner's problem is thus equivalent to finding a solution to a game played by agents that maximize (36).

\subsubsection{Examples}

Let's say agents are firms and each firm has a job that can be either filled (locked in action 1 ) or vacant (locked in action 0 ). Firms with a vacant job choose the search intensity $\delta^{0}$ and matches are destroyed at an exogenous rate $\delta^{1}$, with $c^{1}\left(\delta^{1}\right)=0$ (so $K_{1}$ is a singleton). A vacant job yields a flow payoff $u_{0}\left(\theta_{t}, n_{t}\right)=0$ and a filled job generates a flow payoff $u_{1}\left(\theta_{t}, n_{t}\right)$ that is increasing in both arguments $-\theta_{t}$ can be seen as a measure of productivity and it is assumed that firms' profits are larger when output in the economy (proportional to $n_{t}$ ) is larger.

This is the essence of the model of Howitt and McAfee (1992). They show the existence of multiple equilibria in this setting with no shocks to $\theta$. If $\theta$ follows a Brownian motion, we can apply the results in Frankel and Burdzy (2005), so the model has a unique equilibrium.

The model in Section 3.3 can also be extended to allow for endogenous hazard rates. Firms can be in two regimes: 0 (low capacity) and 1 (high capacity). Firms in regime 1 have some capital that fully depreciate at some exogenous rate. Once it depreciates, firms can decide how much resources (if any) they will spend building a fixed unit of capital (say, constructing a new plant). In other words, they choose the arrival rate of the new plant. The more resources spent, the more likely the new plant will be ready to operate. Guimaraes and Machado (2017) show that the constant-subsidy result also applies to this setting.

\subsection{Ex ante heterogeneous agents}

A consumer deciding between Facebook and another social network takes into account what others have been choosing but also her own tastes; a firm's investment decisions might depend on other firms' investment (which affects aggregate demand), but also on idiosyncratic factors that affect the demand for its particular product. In these and other 
settings, both strategic complementarities and idiosyncratic features of preferences or technologies are important. While strategic complementarities lead agents to try to do what others will be doing, idiosyncratic components of preferences might push agents in different directions. Guimaraes and Pereira (2017) study the interplay of complementarities and heterogeneity in payoffs in a dynamic setting with timing frictions as in Frankel and Pauzner (2000).

Denote agent $i$ 's relative flow-payoff of choosing action 1 by $\Delta u_{q(i)}(\theta, n)$, where $\theta \in \mathbb{R}$ denotes the fundamentals of the economy, $n \equiv \int_{0}^{1} a_{i} d i$ is the fraction of agents currently committed to action 1 , as before, and $q(i) \in \mathcal{Q}=\{1, \ldots, Q\}$ is agent $i$ 's type. All functions $\pi_{q}($.$) are continuously differentiable and strictly increasing in both arguments. If we let$ $\alpha_{q}$ denote the mass of type- $q$ agents in the population and $n_{q}$ the proportion of type- $q$ agents currently playing $1, n$ can be written as $n=\sum_{q=1}^{Q} \alpha_{q} n_{q}$.

An agent who receives a revision opportunity at time $\tau$ will choose $a_{i}=1$ whenever

$$
\mathbb{E} \int_{\tau}^{\infty} e^{-(\rho+\delta)(t-\tau)} \Delta u_{q(i)}\left(\theta_{t}, n_{t}\right) d t>0
$$

and $a_{i}=0$ if the inequality is reversed.

Assumption 1 is now replaced by the assumption that all payoff functions $\Delta u_{q}($.$) are$ such that there are dominance regions for all types of agents. That is, for each $q \in \mathcal{Q}$, there are values $\tilde{\theta}_{q}$ and $\theta_{q}$ such that: if $\theta_{t}>\tilde{\theta}_{q}$, playing 1 is a dominant action, and if $\theta_{t}<\underline{\theta}_{q}$, playing 0 is a dominant action for type- $q$ agents.

In principle, one could expect the dynamics of the system to depend on the proportion of each type of agent currently choosing each option. However, owing to the assumption of a Poisson process for the arrival rate of switching opportunities, we can deal with this problem in a two-dimensional space. Guimaraes and Pereira (2017) show that for any given a strategy profile, the dynamics of $n_{t}$ (i.e., $\partial n_{t} / \partial t$ ) depends on each $n_{q, t}$ (for $q \in \mathcal{Q}$ ) only through $n_{t}$. Denote by $\mathcal{I}_{t}$ the set of types whose strategies prescribe action 1 at time $t$. We have that

$$
\frac{\partial n_{t}}{\partial t}=\delta\left[\sum_{q \in \mathcal{I}_{t}} \alpha_{q}-n_{t}\right]
$$

Guimaraes and Pereira (2017) then show that the uniqueness result in Theorem 1 extends to this environment. Agents choose action 1 when $\theta_{t}>\theta_{q}^{*}\left(n_{t}\right)$ and action 0 when $\theta_{t}<\theta_{q}^{*}\left(n_{t}\right)$, where $\theta_{q}^{*}(\cdot)$ for $q \in \mathcal{Q}$ are decreasing functions. The argument employs a strategy of iterative elimination of strictly dominated strategies similiar to the proof of Theorem 1. 


\subsubsection{Bifurcation probabilities}

From now on, assume there are only two types of agents, $\mathcal{Q}=\{\bar{q}, \underline{q}\}$. Suppose there is a mass $\alpha$ of type- $\bar{q}$ agents, and assume that the utility functions are such that for any pair $(\theta, n)$ we have $\Delta u_{\bar{q}}(\theta, n)>\Delta u_{q}(\theta, n)$, i.e., type- $\bar{q}$ agents have a higher relative instantaneous payoff of choosing action 1 in every state.

The result in Burdzy, Frankel and Pauzner (1998) essentially states that as $\mu, \sigma \rightarrow 0$, the probabilities of the economy bifurcating up and down are proportional to the speed that the economy moves in either direction around the threshold, as in (10). Hence we can extend Burdzy, Frankel and Pauzner's (1998) result on bifurcation probabilities to the case with heterogeneous agents. Suppose agents play according to two arbitrary (downward sloping and Lipschitz) thresholds $\theta_{\bar{q}}(n)<\theta_{\underline{q}}(n)$ for all $n$ in some interval $\left(n^{1}, n^{2}\right)$. Consider a point along one of those thresholds with $n \in\left(n^{1}, n^{2}\right)$. As $\mu, \sigma \rightarrow 0$, the time it takes for the system to bifurcate either up or down converges to zero, the probabilities of an upward or a downward bifurcation are computed as follows:

1. For a point $\left(\theta_{t}, n_{t}\right)$ with $\theta_{t}=\theta_{\bar{q}}\left(n_{t}\right)$,

$$
P(\operatorname{up})= \begin{cases}0 & \text { if } n \geq \alpha, \\ 1-\frac{n}{\alpha} & \text { if } n<\alpha,\end{cases}
$$

and $P($ down $)=1-P($ up $)$.

2. For a point $\left(\theta_{t}, n_{t}\right)$ with $\theta_{t}=\theta_{\underline{q}}\left(n_{t}\right)$.

$$
P(\operatorname{up})= \begin{cases}\frac{1-n}{1-\alpha} & \text { if } n>\alpha, \\ 1 & \text { if } n \leq \alpha,\end{cases}
$$

and $P($ down $)=1-P($ up $)$.

Figure 18: Bifurcation probabilities with two thresholds

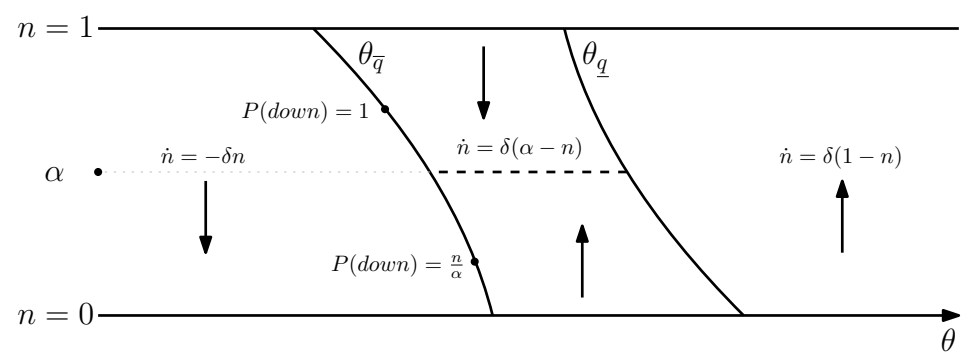

Figure 18 illustrates the dynamics around the two types' thresholds in case they do not intersect (see equation 37) and the implied bifurcation probabilities along the thresholds. 
As in the case with a single threshold, starting somewhere along the curves $\theta_{\bar{q}}$ or $\theta_{\underline{q}}$, once the economy has headed off in one direction, it does not revert to the threshold, since it is downward sloping and shocks to fundamentals are small. The direction of bifurcation depends on the realization of the Brownian motion in a tiny time span and on the speed at which $n$ increases or decreases at each side of the threshold.

To fix ideas, consider a point $\left(\theta_{\bar{q}}(n), n\right)$ with $n>\alpha$. Small negative shocks bring the economy to the left of $\theta_{\bar{q}}$, a region in which $n$ decreases. A tiny shock to the right also puts the economy in a region where $n$ decreases - at a lower speed, but still. ${ }^{16}$ Hence, regardless of the direction of the (small) shocks that hit, the economy immediately heads off in the direction of $n=0$ : the system bifurcates down with probability one. Now, suppose $n<\alpha$. Negative shocks that bring the economy to the left of $\theta_{\bar{q}}$ make $n$ decrease at maximum speed, while positive shocks that lead the economy to the right of the threshold make $n$ increase at rate $\delta(n-\alpha)$.

\subsubsection{Results for limiting cases}

Once again we focus on the tractable limiting cases with vanishing shocks to fundamentals and vanishing timing frictions. Let $\theta_{\bar{q}}^{*}(n)$ and $\theta_{\underline{q}}^{*}(n)$ denote the two types' equilibrium thresholds when $\mu, \sigma \rightarrow 0$. The equilibrium properties in this case depend on the degree of payoff heterogeneity.

Denote by $P_{q}$ the boundary of the upper dominance region of a type-q agent, i.e., the curve along which such agent is indifferent between the two actions if she believes everyone after her will choose 0 (the most pessimistic beliefs possible about the path of $n$ ). Analogously, let $O_{q}$ be the boundary of the lower dominance region for a type-q player - the curve along which the agent is indifferent between 0 and 1 if she believes everyone will choose 1 in the future. If heterogeneity is not too large, dominance regions are such that $O_{\underline{q}}<P_{\bar{q}}(n) \forall n$, so there is a region in which neither action is dominant for both types of agents. Figure 19 illustrates this case.

Proposition 2 shows the main equilibrium properties for the case of vanishing shocks when heterogeneity is not so large. ${ }^{17}$

Proposition 2. In the setting with two types of agents and vanishing shocks, in the unique rationalizable equilibrium, if $\underline{O}(n)<\bar{P}(n) \forall n$, then $\theta_{\bar{q}}^{*}(n)=\theta_{\underline{q}}^{*}(n)$ for all $n$ in an interval containing $\alpha$. Moreover, there are neighborhoods around 0 and 1 in which $\theta_{\bar{q}}^{*}(n)<\theta_{\underline{q}}^{*}(n)$.

\footnotetext{
${ }^{16}$ Between the thresholds, all high-type agents who get the chance will play 1 , but all low-types will play 0 . When $n>\alpha$, there are more agents currently committed to action 1 than agents willing to choose 1 , so $n$ decreases at a rate $\delta(n-\alpha)$.

${ }^{17}$ See Guimaraes and Pereira (2017) for the proof.
} 
Figure 19: The case of not-so-large heterogeneity

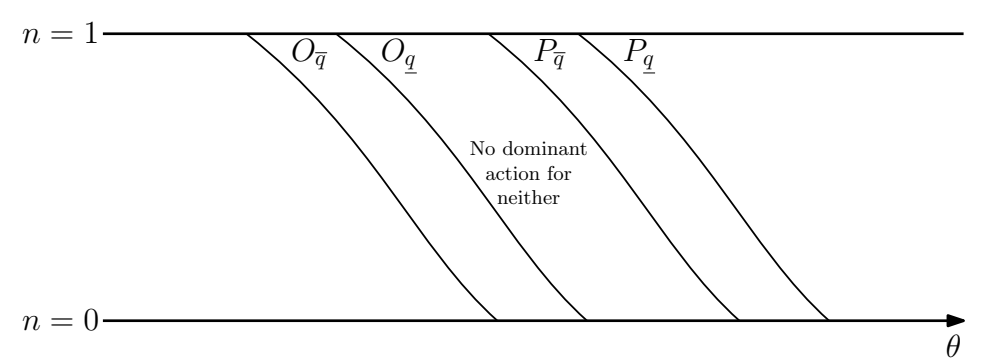

Proposition 2 states that there is some conformity in agents' behavior as long as heterogeneity is not large enough to make it impossible for agents to play according to the same threshold for any (arbitrary) set of beliefs. Moreover, different types choose according to the same threshold for an intermediate range of $n$, but for extreme values of $n$, heterogeneity in preferences beats coordination motives and each type has a distinct threshold. Figure 20 illustrates this result.

Figure 20: Equilibrium with small heterogeneity and $\sigma \rightarrow 0$

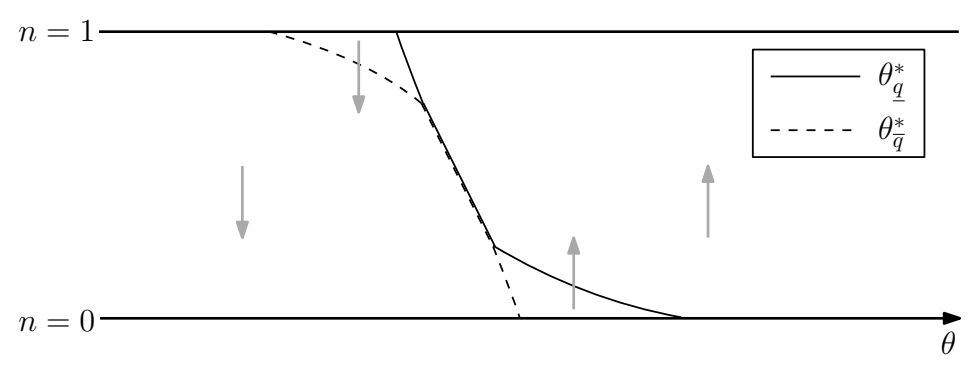

To better understand the result, suppose agents play according to distinct thresholds as in Figure 18. For $n=\alpha$, an agent at the leftmost threshold holds the most pessimistic beliefs, that $n$ will surely decrease from then on at maximum speed. Hence an agent will not choose action 1 unless it is dominant to do so. Conversely, an agent at the rightmost threshold holds the exact opposite beliefs, and thus will not choose 0 unless it is dominant to do so. This reasoning implies that an equilibrium with two distinct thresholds at $n=\alpha$ would require (i) high-type agents being indifferent between either choice for some $\tilde{\theta}$ under the most pessimistic beliefs; and (ii) low-type agents being indifferent between either choice for some $\theta>\tilde{\theta}$ under the most optimistic beliefs, which cannot happen in the case of low heterogeneity. Owing to the large dispersion in beliefs offsetting idiosyncratic payoffs, both thresholds will coincide at $n=\alpha$.

An analogous reasoning helps understand why for extreme values of $n$ thresholds for distinct types do not coincide. Consider $n=0$. Notice beliefs at the two thresholds 
are not so different: both types expect the economy to bifurcate up with probability one. The speed $n$ will move up is not the same in both cases, but that is not such a significant difference in beliefs. That is why even a small difference in preferences leads to the existence of two distinct thresholds.

Consider now the limiting case of $\delta \rightarrow \infty$. The next proposition emphasizes some properties of the equilibrium when distinct types' flow payoffs differ by a constant. ${ }^{18}$

Proposition 3. Let $\Delta u_{\bar{q}}(\theta, n)=\Delta u(\theta, n)+\bar{\varepsilon}$ and $\Delta u_{\underline{q}}(\theta, n)=\Delta u(\theta, n)+\underline{\varepsilon}$, where $\Delta u($. is continuously differentiable and strictly increasing in both arguments and $\bar{\varepsilon}>\underline{\varepsilon}$. Define $\hat{\varepsilon} \equiv \alpha \bar{\varepsilon}+(1-\alpha) \underline{\varepsilon}$ and $\hat{z}^{*}$ as satisfying $\int_{0}^{1} \Delta u\left(\hat{z}^{*}, n\right) d n=-\hat{\varepsilon}$.

In the limit as $\delta \rightarrow \infty, \forall n$, the vertical line $\hat{z}^{*}$ divides the state space in two regions: whenever $\theta_{t}>\hat{z}^{*}, n_{t} \approx 1$ and whenever $\theta_{t}<\hat{z}^{*}, n_{t} \approx 0$.

Proposition 3 states that if heterogeneity is not so large, the economy behaves as if agents were identical and had an intermediate preference parameter $\hat{\varepsilon} \cdot{ }^{19}$ Although agents' strategies do not coincide, agents of a given type immediately switch actions when fundamentals cross the vertical division line, and the change in $n$ leads the opposite type to switch actions as well. Since revision opportunities arrive at a very fast pace, the dynamics of the economy is basically the same as if agents were identical. Figure 21 illustrates the result.

Figure 21: Equilibrium when $\delta \rightarrow \infty$

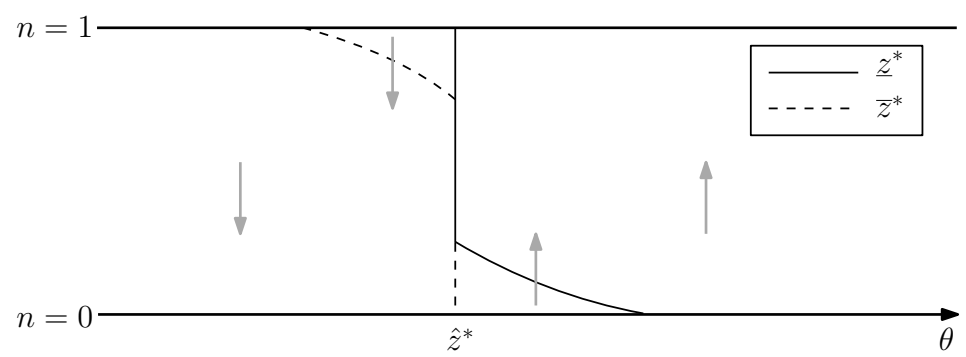

The planner's problem when agents are ex ante heterogeneous can be solved in an analogous way to the one presented in section 2.3. Guimaraes and Pereira (2017) show that the region of the state space where the planner would choose the same strategy for different types is always larger than the region where agents play the same strategy in the decentralized equilibrium. Therefore, from an efficiency perspective, there is not enough conformity. Agents do not internalize the spillovers from their action, and thus do not put enough weight on coordination relative to their own idiosyncratic tastes.

\footnotetext{
${ }^{18}$ For general payoff functions, one can use the bifurcation probabilities to compute the equilibrium analytically.

${ }^{19}$ See Guimaraes and Pereira (2017) for the proof.
} 


\subsection{Coordination and preemption}

We now modify our framework so that agents also worry about preemption. This might be important in applications such as bank runs and currency crises. Consider, for example, a currency pegged to the dollar that might depreciate if enough agents take short positions. While everybody is long, the peg is more likely to be kept, which increases incentives for going long; but if everyone decides to short the currency, the peg is more likely to be abandoned, which raises incentives for agents to go short. This might look like a particular case of a coordination game, but there is an important difference: if agents decide to go short and the peg is likely to be abandoned, one wants to go short before others do it. At this point, there is no coordination game, as agents want to preempt others.

We consider a version of the model in Guimaraes (2006). Agents have 1 unit of resources. An agent that is long in the currency gets return $r$ as long as the peg is kept, so an agent long in the currency for $T$ units of time gets $e^{r T}$, where $r$ is a positive constant. However, when the peg is abandoned, an agent who is long in the currency sees its balances multiply by $e^{-\theta}$. The return for the agents that are not long in the currency are normalized by 0 .

The fundamental variable $\theta$ and the mass of agents long in the currency $n$ determine whether the peg will be abandoned or not. Denote by $\tilde{\theta}$ a function of $n$ that determines when the peg is abandoned. Assume $\tilde{\theta}(0)=0, \tilde{\theta}(1)=\bar{\Theta}$ for some $\bar{\Theta}>0$ and $\tilde{\theta}$ is increasing for $n \in[0, \bar{\Theta}]$. The game is over (and agents long in the currency lose money) whenever $(\theta, n)$ hits $(\tilde{\theta}(n), n)$. For a given $\theta$, the peg is kept as long as $n$ is large enough. Figure 22 provides an illustration.

Figure 22: Game is on if the economy is at the left of $\tilde{\theta}$

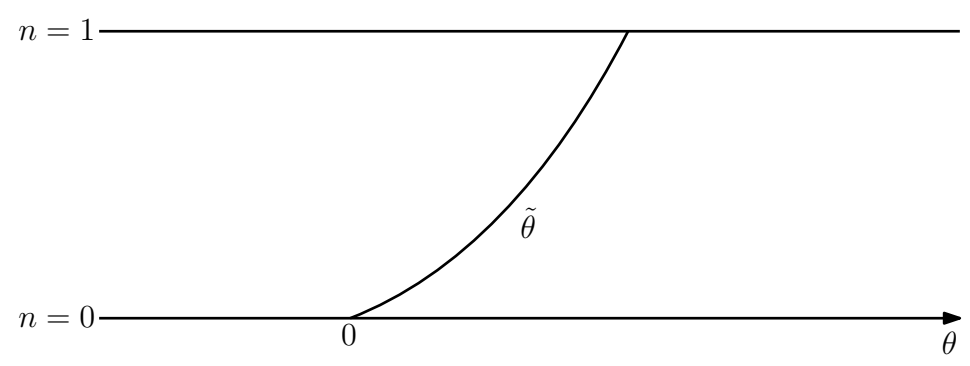

Each agent gets the opportunity to change position according to an independent Poisson process with arrival rate $\delta$, assumed to be greater than $r$. We make the simple assumption that $d \theta_{t}=\mu d t$, for some $\mu>0 .{ }^{20}$

\footnotetext{
${ }^{20}$ Guimaraes (2006) also considers the case where $\theta$ follows a Brownian motion (with a trend).
} 
Related models. Daniëls (2009) studies crises in a model closer to Frankel and Pauzner (2000) and Burdzy, Frankel and Pauzner (2001), but allows for jumps in the fundamental variable $\theta$. In his model, agents actions are always strategic complements.

There are also similarities between the model of debt runs in He and Xiong (2012) and this one. One key difference is that in He and Xiong (2012), there is an exogenous credit line instead of something similar to $n_{t}$, so their economy is not pictured in a twodimensional graph similar to Figures 2 or 22 and the model is solved in a different way.

\subsubsection{Equilibrium}

It can be shown that agents with an opportunity to choose will go long if the economy is at the left of a positively-inclined threshold (call it $\theta^{*}$ ), and will stay out otherwise. Figure 23 shows the decision threshold $\theta^{*}$ and the regions agents choose to go long (L) and decide not to do so $(\mathrm{N})$.

Figure 23: Agents deciding according to $\tilde{\theta}$

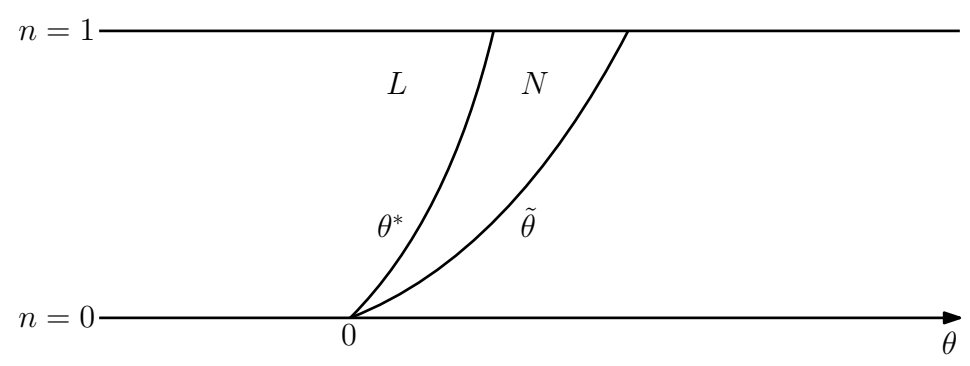

Suppose the economy crosses the threshold $\theta^{*}$ when $n=n_{0}$. Denote by $\tilde{n}$ the value of $n$ when the economy hits the threshold $\tilde{\theta}$ and let $\tilde{\Theta} \equiv \tilde{\theta}(\tilde{n})$. As $\mu>0$, an agent at $\theta^{*}\left(n_{0}\right)$ knows that everybody will choose to stay out from then on. So, the time for a devaluation is related to the fraction of agents that is able to run before the devaluation:

$$
\tilde{n}=n_{0} e^{-\delta \Delta T}
$$

where $\Delta T$ is the time that it takes for the peg to collapse after $\theta$ crosses the threshold.

An agent that is long in the currency sees her resources multiplied by $\exp (r d t)$ at every $d t$ as long as the peg has not been abandoned. However, when the devaluation comes, her balance is multiplied by $\exp (-\theta)$. The only source of uncertainty is the realization of the Poisson process. The expected payoff of a long position is

$$
E \pi=\int_{0}^{\Delta T} \delta e^{-\delta t} e^{r t} d t+e^{r \Delta T} e^{-\tilde{\Theta}} \int_{\Delta T}^{\infty} \delta e^{-\delta t} d t
$$


The first term is what an agent gets if she gets an opportunity to change behavior before time $\Delta T$. The second term is the agent's return if she is caught by the devaluation. An agent is indifferent between a long position and staying out if

$$
E \pi=\left(1-e^{-(\delta-r) \Delta T}\right) \frac{\delta}{\delta-r}+e^{(r-\delta) \Delta T-\tilde{\Theta}}-1
$$

which yields

$$
\Delta T=\frac{\log \left(\frac{\delta}{r}\left(1-e^{-\tilde{\Theta}}\right)+e^{-\tilde{\Theta}}\right)}{\delta-r} .
$$

The expressions in (38) and (39) yield:

$$
\frac{\tilde{n}}{n_{0}}=\left(\frac{r}{r e^{-\tilde{\Theta}}+\delta\left(1-e^{-\tilde{\Theta}}\right)}\right)^{\frac{\delta}{\delta-r}} .
$$

Let $\psi=\frac{\delta}{r}$. Then, (40) can be written as:

$$
\frac{\tilde{n}}{n_{0}}=\left(\psi\left(1-e^{-\tilde{\Theta}}\right)+e^{-\tilde{\Theta}}\right)^{\frac{-\psi}{\psi-1}} .
$$

The expression in (41) and the function $\tilde{\theta}$ determine $\tilde{\Theta}$ and $\tilde{n}$. Then, (38) pins down $\Delta T$. We then need to determine the agents' decision threshold. Let $\Theta^{*} \equiv \theta^{*}\left(n_{0}\right)$. It is pinned down using the expression for the distance covered since agents start to choose to stay out:

$$
\Delta T=\frac{\tilde{\Theta}-\Theta^{*}}{\mu_{\theta}}
$$

Results One implication of (41) is that for a given $\psi, \tilde{\Theta}$ and $\tilde{n}$ do not depend on $\delta$ or $r$. Intuitively, the benefit per unit of time $r$ does not matter; what matters is the benefit per unit of the number of opportunities to run away $r / \delta$.

We now show the following results:

1. $\tilde{\Theta}$ and $\tilde{n}$ are decreasing in $\psi$. Hence $\tilde{\Theta}$ and $\tilde{n}$ are decreasing in $\delta$ and increasing in $r$.

2. As $\psi \rightarrow \infty$,

$$
\lim _{\psi \rightarrow \infty} \tilde{n}=\lim _{\psi \rightarrow \infty} \tilde{\Theta}=\lim _{\psi \rightarrow \infty} \Delta T=\lim _{\psi \rightarrow \infty} \Theta^{*}=0 .
$$

We first prove the first statement. Taking logs from (41) and rearranging yields

$$
\mathcal{F}=\frac{\psi-1}{\psi}\left[\log (\tilde{n})-\log \left(n_{0}\right)\right]+\log \left(\psi\left(1-e^{-\tilde{\Theta}}\right)+e^{-\tilde{\Theta}} \cdot\right)=0
$$

Differentiating $\mathcal{F}$ with respect to $\tilde{n}$ leads to

$$
\frac{\partial \mathcal{F}}{\partial \tilde{n}}=\frac{\psi-1}{\psi \tilde{n}}+\frac{(\psi-1) e^{-\tilde{\Theta}}}{\psi\left(1-e^{-\tilde{\Theta}}\right)+e^{-\tilde{\Theta}}} \frac{\partial \tilde{\theta}}{\partial \tilde{n}}>0 .
$$


Hence, using the implicit function theorem, in order to show that $\tilde{n}$ is decreasing in $\psi$ we need to show that $\mathcal{F}$ is increasing in $\psi$. Differentiating $\mathcal{F}$ with respect to $\psi$, we get

$$
\begin{aligned}
\frac{\partial \mathcal{F}}{\partial \psi} & =\frac{1-e^{-\tilde{\Theta}}}{\psi\left(1-e^{-\tilde{\Theta}}\right)+e^{-\tilde{\Theta}}}-\frac{1}{\psi^{2}}\left[\log \left(n_{0}\right)-\log (\tilde{n})\right] \\
& =\frac{1-e^{-\tilde{\Theta}}}{\psi\left(1-e^{-\tilde{\Theta}}\right)+e^{-\tilde{\Theta}}}-\frac{\log \left(\psi\left(1-e^{-\tilde{\Theta}}\right)+e^{-\tilde{\Theta}}\right)}{\psi(\psi-1)} \\
& >\frac{1-e^{-\tilde{\Theta}}}{\psi\left(1-e^{-\tilde{\Theta}}\right)+e^{-\tilde{\Theta}}}-\frac{\psi\left(1-e^{-\tilde{\Theta}}\right)+e^{-\tilde{\Theta}}-1}{\psi(\psi-1)} \\
& >0
\end{aligned}
$$

The second equality uses (43), the first inequality uses

$$
\log \left(\psi\left(1-e^{-\tilde{\Theta}}\right)+e^{-\tilde{\Theta}}\right)<\psi\left(1-e^{-\tilde{\Theta}}\right)+e^{-\tilde{\Theta}}-1
$$

and the second inequality requires some algebra.

For the second statement, suppose that $\lim _{\psi \rightarrow \infty} \tilde{\Theta}$ is bounded away from 0 . Then from (41), $\lim _{\psi \rightarrow \infty} \tilde{n}=0$, which contradicts the assumptions on the function $\tilde{\theta}$. Hence $\lim _{\psi \rightarrow \infty} \tilde{\Theta}=0$, which implies $\lim _{\psi \rightarrow \infty} \tilde{n}=0$. The results for $\Delta T$ and $\Theta^{*}$ then follow from (39) and (42).

\section{References}

Angeletos, George-Marios, Christian Hellwig, and Alessandro Pavan, 2007, Dynamic global games of regime change: Learning, multiplicity, and the timing of attacks, Econometrica $75,711-756$.

Angeletos, George-Marios, and Chen Lian, 2016, Incomplete information in macroeconomics: Accommodating frictions in coordination, Handbook of Macroeconomics 2, 1065-1240.

Burdzy, Krzysztof, David Frankel, and Ady Pauzner, 1998, On the time and direction of stochastic bifurcation, in Barbara Szyszkowicz, ed., Asymptotic Methods in Probability and Statistics, 483 - 500 (North-Holland, Amsterdam).

Burdzy, Krzysztof, David Frankel, and Ady Pauzner, 2001, Fast equilibrium selection by rational players living in a changing world, Econometrica 69, 163-189.

Carlsson, Hans, and Eric Van Damme, 1993, Global games and equilibrium selection, Econometrica 61, 989-1018. 
Chassang, Sylvain, 2010, Fear of miscoordination and the robustness of cooperation in dynamic global games with exit, Econometrica 78, 973-1006.

Daniëls, Tijmen R, 2009, Unique equilibrium in a dynamic model of speculative attacks, De Economist 157, 417-439.

Dasgupta, Amil, 2007, Coordination and delay in global games, Journal of Economic Theory 134, 195-225.

Dasgupta, Amil, Jakub Steiner, and Colin Stewart, 2012, Dynamic coordination with individual learning, Games and Economic Behavior 74, 83-101.

Frankel, David, and Krzysztof Burdzy, 2005, Shocks and business cycles, Advances in Theoretical Economics 5.

Frankel, David, Stephen Morris, and Ady Pauzner, 2003, Equilibrium selection in global games with strategic complementarities, Journal of Economic Theory 108, 1-44.

Frankel, David, and Ady Pauzner, 2000, Resolving indeterminacy in dynamic settings: The role of shocks, Quarterly Journal of Economics 115, 285-304.

Frankel, David, and Ady Pauzner, 2002, Expectations and the timing of neighborhood change, Journal of Urban Economics 51, 295-314.

Fudenberg, Drew, and Christopher Harris, 1992, Evolutionary dynamics with aggregate shocks, Journal of Economic Theory 57, 420-441.

Guimaraes, Bernardo, 2006, Dynamics of currency crises with asset market frictions, Journal of International Economics 68, 141-158.

Guimaraes, Bernardo, and Caio Machado, 2017, Dynamic coordination and the optimal stimulus policies, Economic Journal forthcoming.

Guimaraes, Bernardo, and Ana Elisa Pereira, 2016, QWERTY is efficient, Journal of Economic Theory 163, 819-825.

Guimaraes, Bernardo, and Ana Elisa Pereira, 2017, Dynamic coordination among heterogeneous agents, Journal of Mathematical Economics forthcoming.

He, Zhiguo, and Wei Xiong, 2012, Dynamic debt runs, Review of Financial Studies 25, 1799-1843.

Howitt, Peter, and R Preston McAfee, 1992, Animal spirits, American Economic Review 82, 493-507. 
Kandori, Michihiro, George J Mailath, and Rafael Rob, 1993, Learning, mutation, and long run equilibria in games, Econometrica 61, 29-56.

Kim, Youngse, 1996, Equilibrium selection inn-person coordination games, Games and Economic Behavior 15, 203-227.

Kiyotaki, Nobuhiro, 1988, Multiple expectational equilibria under monopolistic competition, Quarterly Journal of Economics 103, 695-713.

Kováč, Eugen, and Jakub Steiner, 2013, Reversibility in dynamic coordination problems, Games and Economic Behavior 77, 298-320.

Levin, Jonathan, 2009, The dynamics of collective reputation, The BE Journal of Theoretical Economics 9.

Mathevet, Laurent, and Jakub Steiner, 2013, Tractable dynamic global games and applications, Journal of Economic Theory 148, 2583-2619.

Matsui, Akihiko, and Kiminori Matsuyama, 1995, An approach to equilibrium selection, Journal of Economic Theory 65, 415-434.

Matsuyama, Kiminori, 1991, Increasing returns, industrialization, and indeterminacy of equilibrium, Quarterly Journal of Economics 106, 617-650.

Morris, Stephen, 2014, Coordination, timing and common knowledge, Research in Economics 68, 306-314.

Morris, Stephen, and Hyun Song Shin, 1998, Unique equilibrium in a model of self-fulfilling currency attacks, American Economic Review 587-597.

Morris, Stephen, and Hyun Song Shin, 2003, Global games: Theory and applications, in Mathias Dewatripont, Lars Hansen, and Stephen Turnovsky, eds., Advances in Economics and Econometrics: Theory and Applications, Eighth World Congress, volume 1, Cambridge University Press.

Plantin, Guillaume, and Hyun Song Shin, 2011, Carry trades, monetary policy and speculative dynamics, Manuscript.

Steiner, Jakub, 2008, Coordination cycles, Games and Economic Behavior 63, 308-327. 